EUROPEAN ORGANIZATION FOR NUCLEAR RESEARCH

CERN-PH-EP/2006-028

July 12,2006

\title{
Study of Inclusive Strange-Baryon Production and Search for Pentaquarks in Two-Photon Collisions at LEP
}

The L3 Collaboration

\begin{abstract}
Measurements of inclusive production of the $\Lambda, \Xi^{-}$and $\Xi^{*}(1530)$ baryons in two-photon collisions with the L3 detector at LEP are presented. The inclusive differential cross sections for $\Lambda$ and $\Xi^{-}$are measured as a function of the baryon transverse momentum, $p_{t}$, and pseudo-rapidity, $\eta$. The mean number of $\Lambda, \Xi^{-}$and $\Xi^{*}(1530)$ baryons per hadronic two-photon event is determined in the kinematic range $0.4 \mathrm{GeV}<p_{t}<2.5 \mathrm{GeV},|\eta|<1.2$. Overall agreement with the theoretical models and Monte Carlo predictions is observed. A search for inclusive production of the pentaquark $\theta^{+}(1540)$ in two-photon collisions through the decay $\theta^{+} \rightarrow \mathrm{pK}_{\mathrm{S}}^{0}$ is also presented. No evidence for production of this state is found.
\end{abstract}

Submitted to Eur. Phys. J. C 


\section{Introduction}

Two-photon collisions are the main source of hadron production in high-energy $\mathrm{e}^{+} \mathrm{e}^{-}$interactions at LEP, via the process $\mathrm{e}^{+} \mathrm{e}^{-} \rightarrow \mathrm{e}^{+} \mathrm{e}^{-} \gamma^{*} \gamma^{*} \rightarrow \mathrm{e}^{+} \mathrm{e}^{-}$hadrons, for which the cross section is many orders of magnitude larger than the $\mathrm{e}^{+} \mathrm{e}^{-}$annihilation cross section. The outgoing electron and positron carry almost the full beam energy and their transverse momenta are usually so small that they escape undetected along the beam pipe. At the LEP energies considered here, the negative four-momentum squared of the photons, $Q^{2}$, has an average value of $\left\langle Q^{2}\right\rangle \simeq 0.2 \mathrm{GeV}^{2}$. Therefore, the photons may be considered as "quasi-real". In the Vector Dominance Model (VDM), each virtual photon can fluctuate into a vector meson, thus initiating a strong interaction process with characteristics similar to hadron-hadron interactions. This process dominates in the "soft" interaction region, where hadrons are produced with a low transverse momentum, $p_{t}$. Hadrons with high $p_{t}$ are instead mainly produced by the QED process $\gamma \gamma \rightarrow \mathrm{q} \overline{\mathrm{q}}$ (direct process) or by QCD processes originating from the partonic content of the photon (resolved processes).

Fragmentation mechanisms can be investigated in two-photon reactions. These processes are described phenomenologically. In the Lund string model [1], hadron production proceeds through the creation of quark-antiquark and diquark-antidiquark pairs during string fragmentation. Mesons and baryons are formed by colorless quark-antiquark and quark-diquark combinations, respectively. An extension of this model, the "simple popcorn mechanism" [2], includes the possibility of producing an additional meson between baryon-antibaryon pairs. The relative rate of occurrence of the baryon-meson-antibaryon configuration is governed by the so called "popcorn parameter." Many other parameters must be tuned to reproduce the measured hadron production rate, such as the strange-quark suppression factor, the diquark-to-quark production ratio or the spin-1 diquark suppression factor.

In a statistical model approach [3], hadronisation is described with a reduced number of free parameters. Particles are produced in a purely statistical way from a massive colourless gas which, for hadron production in two-photon collisions, is completely specified by two parameters: the energy density (or temperature) and a strange-quark suppression factor. In this model, the yield of different particles depends only on their masses, spins and quantum numbers.

The L3 Collaboration has previously measured inclusive $\pi^{0}, \mathrm{~K}_{\mathrm{S}}^{0}$ and $\Lambda$ production in quasireal two-photon collisions for a centre-of-mass energy of the two interacting photons, $W_{\gamma \gamma}$, greater than $5 \mathrm{GeV}[4,5]$. In this Article this study is extended to the $\Xi^{-}, \Xi^{*}(1530)$ and $\Omega^{-}$ baryons ${ }^{1)}$. The data sample consists of a total integrated luminosity of $610 \mathrm{pb}^{-1}$, collected with the L3 detector [6] at $\mathrm{e}^{+} \mathrm{e}^{-}$centre-of-mass energies $\sqrt{s}=189-209 \mathrm{GeV}$, with a luminosity weighted average value $\langle\sqrt{s}\rangle=198 \mathrm{GeV}$. Inclusive strange-baryon production has been extensively studied in $\mathrm{e}^{+} \mathrm{e}^{-}$annihilation processes [7], whereas in two-photon collisions only inclusive $\Lambda$ production has been previously measured at lower $\sqrt{s}$ by the TOPAZ Collaboration [8].

Heavy-baryon detection techniques are also applied to search for pentaquark production in two-photon interactions, using the decay channel $\theta^{+} \rightarrow \mathrm{pK}_{\mathrm{S}}^{0}$. Since the first observation of a narrow resonance near $1540 \mathrm{MeV}$ in the $\mathrm{K}^{+} \mathrm{n}$ mass spectrum [9], interpreted as the pentaquark $\theta^{+}$, experimental evidence for and against this new state has been accumulated [10]. Secondgeneration experiments have so far not confirmed the existence of this state [11]. No search in two-photon reactions has been performed yet.

\footnotetext{
${ }^{1)}$ If not stated otherwise, the symbols $\Lambda, \Xi^{-}$and $\Omega^{-}$refer to both the $\Lambda, \Xi^{-}$and $\Omega^{-}$as well as $\bar{\Lambda}, \bar{\Xi}^{+}$and $\bar{\Omega}^{+}$baryons. All charge-conjugate final-states are analysed.
} 


\section{Monte Carlo simulation}

The process $\mathrm{e}^{+} \mathrm{e}^{-} \rightarrow \mathrm{e}^{+} \mathrm{e}^{-}$hadrons is modelled with the PYTHIA [12] and PHOJET [13] event generators with twice the statistics of the data. In PYTHIA, each photon is classified as direct, VDM or resolved, leading to six classes of two-photon events. A smooth transition between these classes is obtained by introducing a $p_{t}$ parameter to specify the boundaries. The SaS1D parametrization is used for the photon parton density function [14]. Since both incoming photons are assumed to be real in the original program, PYTHIA is modified to generate a photon flux according to the equivalent photon approximation [15] with an upper $Q^{2}$ cut at the mass squared of the rho meson.

PHOJET is a general purpose Monte Carlo which describes hadron-hadron, photon-hadron and photon-photon collisions. It relies on the dual parton model combined with the QCDimproved parton model [16]. The $p_{t}$ distribution of the soft partons is matched to the one predicted by QCD to ensure a continuous transition between hard and soft processes. The twophoton luminosity function is calculated in the formalism of Reference 15. The leading-order GRV parametrisation is used for the photon parton density function [17].

In both programs, matrix elements and hard scattering processes are calculated at the leading order and higher-order terms are approximated by a parton shower in the leading-log approximation. The fragmentation is performed within the Lund string fragmentation scheme as implemented in JETSET [12], which is also used to simulate the hadronisation process. JETSET parameters are tuned by using hadronic Z decays [18]. In particular, the strangenesssuppression factor is set to 0.3, whereas the parameter governing the extra suppression of strange quarks in diquarks is fixed to 0.4 and the diquark-to-quark production ratio to 0.10. The popcorn parameter is set to 0.5 and a value $\alpha_{S}\left(m_{\mathrm{Z}}\right)=0.12$ is used for the strong couplingconstant.

The following Monte Carlo generators are used to simulate the background processes: KK2f [19] for the annihilation process $\mathrm{e}^{+} \mathrm{e}^{-} \rightarrow \mathrm{q} \overline{\mathrm{q}}(\gamma)$; KORALZ [20] for $\mathrm{e}^{+} \mathrm{e}^{-} \rightarrow \tau^{+} \tau^{-}(\gamma)$; KORALW [21] for $\mathrm{e}^{+} \mathrm{e}^{-} \rightarrow \mathrm{W}^{+} \mathrm{W}^{-}$and DIAG36 [22] for $\mathrm{e}^{+} \mathrm{e}^{-} \rightarrow \mathrm{e}^{+} \mathrm{e}^{-} \tau^{+} \tau^{-}$. The response of the L3 detector is simulated using the GEANT [23] and GHEISHA [24] programs. Timedependent detector inefficiencies, as monitored during each data-taking period, are included in the simulations. All simulated events are passed through the same reconstruction program as the data.

\section{Two-photon event selection}

Two-photon interaction events are mainly collected by track triggers [25], with a track $p_{t}$ threshold of about $150 \mathrm{MeV}$, and the calorimetric energy trigger [26]. The selection of $\mathrm{e}^{+} \mathrm{e}^{-} \rightarrow$ $\mathrm{e}^{+} \mathrm{e}^{-}$hadrons events [27] is based on information from the central tracking detectors and the electromagnetic and hadronic calorimeters. It consists of:

- A multiplicity cut. To select hadronic final states, at least six objects must be detected, where an object can be a track satisfying minimal quality requirements or an isolated cluster in the BGO electromagnetic calorimeter of energy greater than $100 \mathrm{MeV}$.

- Energy cuts. The total energy deposited in the calorimeters must be less than $40 \%$ of $\sqrt{s}$ to suppress events from the $\mathrm{e}^{+} \mathrm{e}^{-} \rightarrow \mathrm{q} \overline{\mathrm{q}}(\gamma)$ and $\mathrm{e}^{+} \mathrm{e}^{-} \rightarrow \tau^{+} \tau^{-}(\gamma)$ processes. In addition, the total energy in the electromagnetic calorimeter is required to be greater than $500 \mathrm{MeV}$ 
to suppress beam-gas and beam-wall interactions and less than $50 \mathrm{GeV}$ to remove events from the annihilation process $\mathrm{e}^{+} \mathrm{e}^{-} \rightarrow \mathrm{q} \overline{\mathrm{q}}(\gamma)$.

- An anti-tag condition. Events containing a cluster in the luminosity monitor with an electromagnetic shower shape, and energy greater than $30 \mathrm{GeV}$, are excluded from the analysis. The luminosity monitor covers the polar angular region $31 \mathrm{mrad}<\theta<62 \mathrm{mrad}$ on both sides of the detector. In addition, events with an electron or positron scattered above $62 \mathrm{mrad}$ are rejected by the cut on calorimetric energy.

- A mass cut. The mass of all visible particles, $W_{v i s}$, must be greater than $5 \mathrm{GeV}$ to exclude the resonance region. In this calculation, the pion mass is attributed to tracks while isolated electromagnetic clusters are treated as massless.

About 3 million hadronic events are selected by these criteria with an overall efficiency of $45 \%$ for a two-photon centre-of-mass energy, $W_{\gamma \gamma}$, greater than $5 \mathrm{GeV}$. The background level of this sample is less than $1 \%$ and is mainly due to the $\mathrm{e}^{+} \mathrm{e}^{-} \rightarrow \mathrm{q} \overline{\mathrm{q}}(\gamma)$ and $\mathrm{e}^{+} \mathrm{e}^{-} \rightarrow \mathrm{e}^{+} \mathrm{e}^{-} \tau^{+} \tau^{-}$ processes. The backgrounds from beam-gas and beam-wall interactions are negligible.

\section{Strange baryon selection}

The $\Lambda, \Xi^{-}$and $\Omega^{-}$baryons are identified through the decays $\Lambda \rightarrow \mathrm{p} \pi^{-}, \Xi^{-} \rightarrow \Lambda \pi^{-}$and $\Omega^{-} \rightarrow$ $\Lambda \mathrm{K}^{-}$. Due to their long lifetimes, the combinatorial background can be strongly suppressed by selecting secondary vertices which are clearly displaced from the interaction point. The $\Xi^{*}(1530)$ decays strongly into $\Xi^{-} \pi^{+}$and no corresponding displaced vertex can be observed.

The $\Xi^{-}, \Omega^{-}$and $\Xi^{*}(1530)$ final states are reconstructed by combining $\Lambda$ candidates with pion or kaon candidates. The latter are defined as tracks formed by at least 30 hits in the central tracker out of a maximum of 62 and a $p_{t}>100 \mathrm{MeV}$. The probability of the pion or kaon hypothesis, based on the $\mathrm{d} E / \mathrm{d} x$ measurement in the tracking chamber, is required to be greater than 0.01 .

\section{1 $\Lambda$ selection}

The selection procedure is unchanged from our previous publication [5]. The $\Lambda$ identification is optimized to achieve a high efficiency and a good background suppression by selecting secondary decay vertices satisfying the following conditions:

- The distance $d_{\Lambda}$ in the transverse plane $\mathrm{e}^{2)}$ between the secondary vertex and the $\mathrm{e}^{+} \mathrm{e}^{-}$ interaction point must be greater than $3 \mathrm{~mm}$.

- The angle $\alpha_{\Lambda}$ between the sum $p_{t}$ vector of the two tracks and the direction in the transverse plane between the $\mathrm{e}^{+} \mathrm{e}^{-}$interaction point and the secondary vertex must be less than 100 mrad.

The distributions of these variables are presented in Figure1. Good agreement between data and Monte Carlo is observed. The proton is identified as the track with the largest momentum, an assignment shown by Monte Carlo to be correct more than $99 \%$ of the time. The $\mathrm{d} E / \mathrm{d} x$

\footnotetext{
${ }^{2)}$ The transverse plane is defined as the plane transverse to the beam direction.
} 
measurements in the central tracking chamber must be consistent with this assignment, a probability greater than 0.01 for both the proton and the pion candidates being required.

The distribution of the corresponding invariant mass of the $\mathrm{p} \pi$ system, $m(\mathrm{p} \pi)$, is shown in Figure 2a. A clear $\Lambda$ peak over a smooth background is visible, compatible with the $\Lambda$ baryon mass, $m_{\Lambda}=1115.68 \pm 0.01 \mathrm{MeV}$ [7]. The resolution of the signal, about $3 \mathrm{MeV}$, is well reproduced by Monte Carlo simulation. The $m(\mathrm{p} \pi)$ mass spectrum for the $\mathrm{p} \pi^{-}$and $\overline{\mathrm{p}} \pi^{+}$ combinations are shown in Figure $2 \mathrm{~b}$ and $2 \mathrm{k}$, respectively. The numbers of $\Lambda$ baryons for different $p_{t}$ and $|\eta|$ bins are given in Tables 1 and 2. They are determined by a fit in which the signal is modelled by a Gaussian and the background by a fourth-order Chebyshev polynomial. Consistent values of the fitted mass and width are found for the different $p_{t}$ and $|\eta|$ bins.

\section{$4.2 \Xi^{-}$selection}

The $\Xi^{-}$baryons are reconstructed by combining $\Lambda$ candidates with pion candidates. For the identification of $\Xi^{-}$and $\Omega^{-}$baryons, different criteria are used to select secondary vertices produced by $\Lambda$ decays. The distance $d_{\Lambda}$ is required to be greater than $5 \mathrm{~mm}$ and the angle $\alpha_{\Lambda}$ less than $200 \mathrm{mrad}$, as shown in Figure $3 \mathrm{a}$ and $3 \mathrm{~b}$. The other cuts are left unchanged. The distribution of the resulting invariant mass $m(\mathrm{p} \pi)$ is displayed in Figure $3 \mathrm{k}$ and shows a clear $\Lambda$ peak. The $76000 \mathrm{p} \pi$ combinations that lie in the mass interval $1.105 \mathrm{GeV}<m(\mathrm{p} \pi)<1.125 \mathrm{GeV}$ are retained. To reduce the combinatorial background, the following criteria are applied:

- the distance of closest approach (DCA) in the transverse plane of the $\Xi^{-}$decay pion to the $\mathrm{e}^{+} \mathrm{e}^{-}$interaction point, $d_{\pi}$, must be greater than $1 \mathrm{~mm}$.

- the distance in the transverse plane between the $\Lambda \pi$ vertex and the $\mathrm{e}^{+} \mathrm{e}^{-}$interaction point, $d_{\Xi}$, is required to be greater than $d_{\Lambda}$.

- the angle $\alpha_{\Xi}$ between the $p_{t}$ vector of the $\Lambda \pi$ combination and the direction in the transverse plane between the $\mathrm{e}^{+} \mathrm{e}^{-}$interaction point and the $\Lambda \pi$ vertex has to be less than 100 mrad.

Distributions of the difference $d_{\Lambda}-d_{\Xi}$ and of the angle $\alpha_{\Xi}$ are displayed in Figure 4 and exhibit a good agreement with the Monte Carlo predictions.

The distribution of the mass of the $\Lambda \pi$ system, $m(\Lambda \pi)$, is displayed in Figure 5 for the $\Lambda \pi^{-}$ and $\bar{\Lambda} \pi^{+}$combinations, respectively. Figure 6 shows the $m(\Lambda \pi)$ spectrum for the different $p_{t}$ bins listed in Table 1. A clear $\Xi^{-}$peak is visible over a smooth background, compatible with the measured $\Xi^{-}$mass, $m_{\Xi^{-}}=1321.31 \pm 0.13 \mathrm{MeV}$ [7]. The resolution of $m(\Lambda \pi)$, about $7 \mathrm{MeV}$, is well reproduced by Monte Carlo simulation. The number of $\Xi^{-}$baryons in each $p_{t}$ and $|\eta|$ bin is evaluated by means of a fit to the $m(\Lambda \pi)$ spectrum in the interval $1.26 \mathrm{GeV}<m(\mathrm{p} \pi)<1.4 \mathrm{GeV}$. The signal is modelled with a Gaussian and the background by a fourth-order Chebyshev polynomial. The results are listed in Tables 1 and 2 .

\section{3 $\Xi^{*}(1530)$ selection}

The $\Xi^{*}(1530)$ baryons are identified by reconstructing the decays $\Xi^{*}(1530) \rightarrow \Xi^{-} \pi^{+}$and $\Xi^{*}(1530) \rightarrow \Xi^{+} \pi^{-}$. The $\Xi^{-}$candidates with a mass of $\pm 15 \mathrm{MeV}$ around the nominal $\Xi^{-}$ mass are combined with pion candidates. As the latter are produced at the $\mathrm{e}^{+} \mathrm{e}^{-}$interaction point, their DCA must be less than $5 \mathrm{~mm}$. To compare the $\Xi^{*}(1530)$ production to other strange 
baryons, the measurement is restricted to the kinematical region: $0.4 \mathrm{GeV}<p_{t}<2.5 \mathrm{GeV}$, $|\eta|<1.2$. The distribution of the invariant mass of the $\Xi \pi$ system, $m(\Xi \pi)$, is shown in Figure Th for opposite-charge $\left(\Xi^{-} \pi^{+}\right.$and $\left.\bar{\Xi}^{+} \pi^{-}\right)$and same-charge $\left(\Xi^{-} \pi^{-}\right.$and $\left.\bar{\Xi}^{+} \pi^{+}\right)$combinations. The number of same-charge combinations is normalized to that of opposite-charge combinations in the region $m(\Xi \pi)>1.7 \mathrm{GeV}$. An excess corresponding to the $\Xi^{*}(1530)$ is observed in the opposite-charge spectrum close to the nominal mass $m_{\Xi^{*}(1530)}=1531.80 \pm 0.32 \mathrm{MeV}[7]$. The number of $\Xi^{*}(1530)$ baryons is determined by means of a fit to the mass spectrum in the region $1.47 \mathrm{GeV}<m(\Xi \pi)<1.90 \mathrm{GeV}$, as shown in Figure $7 \mathrm{~b}$. The signal is modelled with a Gaussian and the background is parametrized by a threshold function of the form:

$$
a\left(m_{(\Xi \pi)}-m_{0}\right)^{b} \exp \left[c\left(m_{(\Xi \pi)}-m_{0}\right)+d\left(m_{(\Xi \pi)}-m_{0}\right)^{2}\right]
$$

where $a, b, c, d$ and $m_{0}$ are free parameters. The results are given in Table 1 .

\subsection{Search for $\Omega^{-}$}

Since the topology of the $\Omega^{-} \rightarrow \Lambda \mathrm{K}^{-}$decay is similar to that of $\Xi^{-} \rightarrow \Lambda \pi^{-}$, the selection criteria are identical except that kaon candidates are combined with $\Lambda$ candidates instead of pion candidates. The corresponding $m(\Lambda \mathrm{K})$ spectrum is displayed in Figure 8 , No signal is observed around the nominal mass of the $\Omega^{-}$baryon, $m_{\Omega^{-}}=1672.45 \pm 0.29 \mathrm{MeV}$ [7]. The expected signal, as predicted by PHOJET, is found to be almost negligible.

\section{$5 \quad$ Results and systematic uncertainties}

The cross sections for $\Lambda, \Xi^{-}$and $\Xi^{*}(1530)$ production are measured for $W_{\gamma \gamma}>5 \mathrm{GeV}$, with a mean value $\left\langle W_{\gamma \gamma}\right\rangle=30 \mathrm{GeV}$, and a photon virtuality $Q^{2}<8 \mathrm{GeV}^{2}$ with $\left\langle Q^{2}\right\rangle \simeq 0.2 \mathrm{GeV}^{2}$. This kinematical region is defined by cuts at Monte Carlo generator level.

The overall efficiencies for detecting $\Lambda, \Xi^{-}$and $\Xi^{*}(1530)$ baryons as a function of $p_{t}$ and $|\eta|$ are listed in Tables 1 and 2. They include reconstruction and trigger efficiencies as well as branching fractions for the decays $\Xi^{*}(1530) \rightarrow \Xi^{-} \pi^{+}, \Xi^{-} \rightarrow \Lambda \pi^{-}$and $\Lambda \rightarrow \mathrm{p} \pi^{-}: 67 \%$, $100 \%$ and $64 \%$, respectively [7]. The reconstruction efficiencies, which include effects of the acceptance and the selection cuts, are calculated with the PHOJET and PYTHIA Monte Carlo generators. As both generators reproduce well the shapes of the experimental distributions of hadronic two-photon production [27], the average selection efficiency is used. The track-trigger efficiency is calculated for each data-taking period by comparing the number of events accepted by the track triggers and the independant calorimetric-energy triggers. The efficiency of the higher level triggers is measured using prescaled events. The total trigger efficiency varies from $82 \%$ for $p_{t}<0.4 \mathrm{GeV}$ to $85 \%$ in the high $p_{t}$ region.

The differential cross sections $\mathrm{d} \sigma / \mathrm{d} p_{t}, \mathrm{~d} \sigma / \mathrm{d} p_{t}^{2}$ and $\mathrm{d} \sigma / \mathrm{d}|\eta|$ for the reactions $\mathrm{e}^{+} \mathrm{e}^{-} \rightarrow \mathrm{e}^{+} \mathrm{e}^{-} \Lambda \mathrm{X}$ and $\mathrm{e}^{+} \mathrm{e}^{-} \rightarrow \mathrm{e}^{+} \mathrm{e}^{-} \Xi^{-} \mathrm{X}$ are given in Table 1 and Table 2, respectively. The mean number of $\Lambda$, $\Xi^{-}$and $\Xi^{*}(1530)$ baryons per hadronic two-photon event is measured in the region $0.4 \mathrm{GeV}<$ $p_{t}<2.5 \mathrm{GeV},|\eta|<1.2$ and $W_{\gamma \gamma}>5 \mathrm{GeV}$, as summarized in Table 3 Finally, the ratio of $\Lambda$ to $\bar{\Lambda}$ and $\Xi^{-}$to $\bar{\Xi}^{+}$baryons are determined from the respective mass spectra. They are found to be $\mathrm{N}(\bar{\Lambda}) / \mathrm{N}(\Lambda)=0.99 \pm 0.04$ and $\mathrm{N}\left(\bar{\Xi}^{+}\right) / \mathrm{N}\left(\Xi^{-}\right)=0.96 \pm 0.14$, where the uncertainties are statistical. These results are in agreement with the value of 1.0 expected for baryon pair production in two-photon reactions.

The following sources of systematic uncertainties are considered: selection procedure, background subtraction, limited Monte Carlo statistics, Monte Carlo modelling and the accuracy 
of the trigger efficiency measurement. Their contributions to the cross section measurements are detailed in Table 4 .

The uncertainties associated to the selection criteria are evaluated by changing the corresponding cuts and repeating the fitting procedure. The $\Lambda$ selection uncertainty is dominated by the secondary vertex identification while the largest uncertainty for the $\Xi^{-}$channel, about $9 \%$, arises from the $\Lambda \pi$ vertex reconstruction. The uncertainty due to the $\mathrm{e}^{+} \mathrm{e}^{-} \rightarrow \mathrm{e}^{+} \mathrm{e}^{-}$hadrons event selection is less than $1 \%$.

The uncertainty due to background subtraction is assessed by using different background parameterizations and fit intervals in the fitting procedure. The Monte Carlo modelling uncertainty, taken as half the relative difference difference between PHOJET and PYTHIA, varies between $1 \%$ and $14 \%$ whereas the uncertainty associated to the limited Monte Carlo statistics varies from $1 \%$ for $\Lambda$ to $18 \%$ for $\Xi^{*}(1530)$. A systematic uncertainty of $2 \%$ is assigned to the determination of the trigger efficiency, which takes into account the determination procedure and time stability.

\section{Comparison with Monte Carlo and theoretical models}

The differential cross sections $\mathrm{d} \sigma / \mathrm{d} p_{t}^{2}\left(\mathrm{e}^{+} \mathrm{e}^{-} \rightarrow \mathrm{e}^{+} \mathrm{e}^{-} \Lambda \mathrm{X}\right)$ and $\mathrm{d} \sigma / \mathrm{d} p_{t}^{2}\left(\mathrm{e}^{+} \mathrm{e}^{-} \rightarrow \mathrm{e}^{+} \mathrm{e}^{-} \Xi^{-} \mathrm{X}\right)$ for $|\eta|<1.2$ are presented in Figure 9a. The behaviour of the cross sections is well described by an exponential of the form $A \exp \left(-a p_{t}^{2}\right)$ in the region $0.16 \mathrm{GeV}^{2}<p_{t}^{2}<1.69 \mathrm{GeV}^{2}$ with $a=1.78 \pm 0.16 \mathrm{GeV}^{-2}$ and $a=1.68 \pm 0.35 \mathrm{GeV}^{-2}$ for $\Lambda$ and $\Xi^{-}$production, respectively. These two values are compatible with each other, as expected from the Lund string model. The region $1.69 \mathrm{GeV}^{2}<p_{t}^{2}<6.25 \mathrm{GeV}^{2}$ is better reproduced by a power law of the form $A p_{t}^{-b}$ with $b=5.2 \pm 0.2$ and $b=4.2 \pm 0.9$ for $\Lambda$ and $\Xi^{-}$baryons, respectively.

The differential cross sections $\mathrm{d} \sigma / \mathrm{d} p_{t}\left(\mathrm{e}^{+} \mathrm{e}^{-} \rightarrow \mathrm{e}^{+} \mathrm{e}^{-} \Lambda \mathrm{X}\right)$ and $\mathrm{d} \sigma / \mathrm{d} p_{t}\left(\mathrm{e}^{+} \mathrm{e}^{-} \rightarrow \mathrm{e}^{+} \mathrm{e}^{-} \Xi^{-} \mathrm{X}\right)$ for $|\eta|<1.2$ are displayed in Figure 9b. Phase-space suppression explains the lower values obtained in the first bins. The behaviour of the cross sections is well described by an exponential of the form $A \exp \left(-p_{t} /\left\langle p_{t}\right\rangle\right)$ in the region $0.75 \mathrm{GeV}<p_{t}<2.5 \mathrm{GeV}$ with a mean value $\left\langle p_{t}\right\rangle=368 \pm$ $17 \mathrm{MeV}$ for $\Lambda$ production and in the range $0.7 \mathrm{GeV}<p_{t}<2.5 \mathrm{GeV}$ with $\left\langle p_{t}\right\rangle=472 \pm 98 \mathrm{MeV}$ for $\Xi^{-}$formation. These values are larger than those obtained for inclusive $\pi^{0}$ and $K_{\mathrm{S}}^{0}$ production: $\left\langle p_{t}\right\rangle \simeq 230 \mathrm{MeV}$ and $\left\langle p_{t}\right\rangle \simeq 290 \mathrm{MeV}$, respectively [4].

The data are compared to the PHOJET and PYTHIA Monte Carlo predictions in Figure 10a. Both Monte Carlo programs fail to reproduce the shape and the normalization of the $\Lambda$ cross section. A better agreement can be achieved by adjusting the width $\sigma_{q}$ in the Gaussian $p_{t}$ distribution for primary hadrons produced during the fragmentation. As shown in Figure 10 $\mathrm{b}$, the predictions obtained with PYTHIA for $\Lambda$ production using a value $\sigma_{q}=0.546 \mathrm{GeV}$ reproduce much better the data than those with the default value $\sigma_{q}=0.411 \mathrm{GeV}$. On the other hand, an increase of the width of the $p_{t}$ distribution of quarks inside initial photons does not improve the description of the measured spectra. Similar trends are observed with PHOJET.

The differential cross sections as a function of $|\eta|$ are displayed in Figure 11] and 11b. Both Monte Carlo programs describe well the almost-uniform $|\eta|$ shape, while the size of the discrepancy on the absolute normalization depends on the $p_{t}$ range.

The mean numbers of $\Lambda, \Xi^{-}$and $\Xi^{*}(1530)$ baryons per hadronic two-photon event are extrapolated from the measured phase-space to the full $p_{t}$ and $|\eta|$ range using the PYTHIA Monte Carlo with the adjusted value $\sigma_{q}=0.546 \mathrm{GeV}$. The uncertainty on the extrapolation factors propagates to the results as an additional systematic uncertainty. It arises mainly from 
the modelling of the two-photon reaction and fragmentation processes. The contribution due to fragmentation, about $7 \%$, is estimated using an independent fragmentation model [28] instead of the Lund string scheme to simulate baryon production as well as varying the parameters of these models. The two-photon modelling uncertainty, taken as the difference between the extrapolation factors estimated by PYTHIA and PHOJET, is found to be $3 \%$. The results are summarized in Table 3 together with the predictions of PYTHIA and PHOJET. Both Monte Carlos overestimate the $\Lambda$ and $\Xi^{-}$mean numbers, the discrepancy being less pronounced in PYTHIA than in PHOJET. A better agreement is obtained by decreasing the strange-quark suppression factor by a few percent. These extrapolated mean numbers can be converted to inclusive cross sections by multiplying them for the total two-photon hadronic cross section measured with the same detector as discussed in Reference [27].

The predictions of the thermodynamical model of Reference 3 are displayed in Figure 12 as a function of the strangeness suppression factor $\gamma_{s}$, using as energy density the value $\rho=0.4[3]$. Overall agreement is observed for a $\gamma_{s}$ in the vicinity of $\gamma_{s}=0.7$, which is similar to the value extracted from $\mathrm{e}^{+} \mathrm{e}^{-}$annihilation events [29].

As suggested in Reference 30, the mean numbers $\langle n\rangle$ of octet and decuplet baryons can also be parametrized by a function of the form:

$$
\langle n\rangle=a[(2 J+1) /(2 I+1)] \exp \left(-b m^{2}\right)
$$

where $I, J, m$ are respectively the isospin, total angular momentum and mass of the baryons and $a, b$ are free parameters. The ratios $[(2 J+1) /(2 I+1)]\langle n\rangle$ are displayed in Figure [13] together with the data measured in $\mathrm{e}^{+} \mathrm{e}^{-}$collisions at $\sqrt{s}=10 \mathrm{GeV}$ and $\sqrt{s}=91 \mathrm{GeV}[7]$. A fit to our data gives $b=4.5 \pm 0.9$, a value compatible with the exponents $b=4.3 \pm 0.6$ and $b=4.0 \pm 0.1$ obtained for $\mathrm{e}^{+} \mathrm{e}^{-}$reactions at $\sqrt{s}=10 \mathrm{GeV}$ and $\sqrt{s}=91 \mathrm{GeV}$, respectively. This provides evidence for the universality of fragmentation processes in two-photon and $\mathrm{e}^{+} \mathrm{e}^{-}$ reactions.

\section{Search for the $\theta^{+}$pentaquark}

A $\theta^{+3)}$ Monte Carlo consisting of pentaquarks mixed with large samples of hadronic twophoton events is used to study the kinematics of the $\theta^{+}$decay products and estimate the signal efficiency. In this Monte Carlo the pentaquarks are generated with a mass of $1.54 \mathrm{GeV}$ and a width of $1 \mathrm{MeV}$. The transverse momentum and pseudo-rapidity distributions predicted by the PYTHIA event generator for inclusive $\Xi^{*}(1530)$ production are taken to model the unknown distributions of the pentaquarks. As these distributions are very similar for different low mass baryons, the simulated acceptance is expected to be quite insensitive to the choice of a particular baryon. The $\theta^{+} \rightarrow \mathrm{pK}_{\mathrm{S}}^{0}$ decay is assumed to be isotropic. The $\mathrm{K}_{\mathrm{S}}^{0} \mathrm{p}$ and $\mathrm{K}_{\mathrm{S}}^{0} \overline{\mathrm{p}}$ mass distributions, calculated at the generator level, show no evidence of a narrow peak close to $1540 \mathrm{MeV}$. This indicates that no reflections from known decay modes could generate a fake $\theta^{+}$signal.

\subsection{Event selection}

The $\theta^{+}$candidates are reconstructed using the decay $\theta^{+} \rightarrow \mathrm{pK}_{\mathrm{S}}^{0}$. Events are selected if they contain at least one track coming from the $\mathrm{e}^{+} \mathrm{e}^{-}$interaction point and a $\mathrm{K}_{\mathrm{S}}^{0}$ decaying into $\pi^{+} \pi^{-}$ at a secondary vertex. The $\mathrm{K}_{\mathrm{S}}^{0}$ selection proceeds as follows:

\footnotetext{
${ }^{3)}$ If not stated otherwise, the symbols $\theta^{+}$refers to $\theta^{+}$and $\bar{\theta}^{-}$as both charge-conjugate final-states are analysed.
} 
- Each track is required to have more than 12 hits out a maximum of 62 and $p_{t}>100 \mathrm{MeV}$.

- The $\mathrm{d} E / \mathrm{d} x$ measurement of both pion candidates must be consistent with this hypothesis with a probability greater than 0.01 .

- The distance in the transverse plane between the secondary vertex and the $\mathrm{e}^{+} \mathrm{e}^{-}$interaction point must be greater than $5 \mathrm{~mm}$.

- The angle between the sum $p_{t}$ vector of the two tracks and the direction in the transverse plane between the primary interaction point and the secondary vertex must be less than 75 mrad.

The distribution of the effective mass of the $\pi \pi$ system, $m(\pi \pi)$, is shown in Figure 14. A clear $\mathrm{K}_{\mathrm{S}}^{0}$ peak is present over a smooth background. The spectrum is fitted with two Gaussian

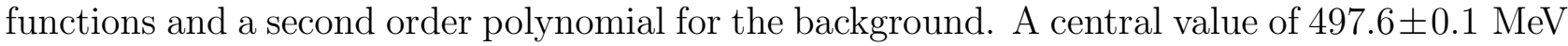
is obtained for the peak, which agrees with the expected value of $497.7 \mathrm{MeV}$ [7]. To search for $\theta^{+}$, the $\mathrm{K}_{\mathrm{S}}^{0}$ candidates are selected within an interval of $\pm 20 \mathrm{MeV}$ around the central value of the peak, corresponding to $140000 \mathrm{~K}_{\mathrm{S}}^{0}$ candidates with a purity of $69 \%$.

The proton and antiproton candidates are tracks with at least 30 hits out of a maximum of 62 and a DCA less than $5 \mathrm{~mm}$. In addition, the $\mathrm{d} E / \mathrm{d} x$ measurement in the central tracker must be consistent with the proton hypothesis with a probability greater than 0.05 . To reject kaons and pions, the probability of each of these hypothesis must be less than 0.01 . A total of 7131 selected protons and 5340 antiprotons are selected. Their $\mathrm{d} E / \mathrm{d} x$ measurement is shown in Figure 15 together with theoretical the expectations based on the Bethe-Bloch formula. The purity of the selected sample is greater than $96 \%$. The excess of protons, due to secondary interactions between particles and the detector, is well reproduced by the Monte Carlo.

The resulting $\mathrm{pK}_{\mathrm{S}}^{0}$ and $\overline{\mathrm{p}} \mathrm{S}_{\mathrm{S}}^{0}$ mass spectra, $m\left(\mathrm{pK}_{\mathrm{S}}^{0}\right)$, are shown in Figure 16a and 16 b respectively. Since no distinct structure due to the excess of protons is observed, the two spectra are combined in the following.

\subsection{Results}

An upper limit for the number of $\mathrm{e}^{+} \mathrm{e}^{-} \rightarrow \mathrm{e}^{+} \mathrm{e}^{-} \theta^{+} \mathrm{X}$ events at $95 \%$ confidence level is derived with a fit to the $m\left(\mathrm{pK}_{\mathrm{S}}^{0}\right)$ mass spectrum. To minimize the effect of the binning and the choice of the fitting interval, an unbinned maximum likelihood fit is performed in the range $1.45 \mathrm{GeV}<W_{\gamma \gamma}<1.8 \mathrm{GeV}$. The likelihood function is written as:

$$
\mathcal{L}=p \cdot g\left(m_{\left(\mathrm{pK}_{\mathrm{S}}^{0}\right)}\right)+(1-p) \cdot b\left(m_{\left(\mathrm{pK}_{\mathrm{S}}^{0}\right)}\right)
$$

where $p$ denotes the fraction of signal events inside the fitted region. The background, $b\left(m_{\left(\mathrm{pK}_{\mathrm{S}}^{0}\right)}\right)$, is parametrized by a fourth-order polynomial and the signal by a Gaussian distribution, $g\left(m_{\left(\mathrm{pK}_{\mathrm{S}}^{0}\right)}\right)$. The resolution of the expected signal is determined from Monte Carlo to be $14.0 \pm 0.6 \mathrm{MeV}$. The resulting fit is displayed in Figure 17 and yields a fraction of signal events compatible with zero: $p=-0.006 \pm 0.007$. Restricting the measurement to the physical region $p \geq 0$, an upper limit of 59.3 events at $95 \%$ confidence level is derived.

The $\theta^{+}$selection efficiency is estimated to be $1.0 \%$, including a branching fraction $\mathrm{K}_{\mathrm{S}}^{0} \rightarrow$ $\pi^{+} \pi^{-}$of $69 \%$ [7]. The branching ratio $\theta^{+} \rightarrow \mathrm{pK}_{\mathrm{S}}^{0}$ is set to $1 / 4$, accounting for competition with both the $\mathrm{K}^{+} \mathrm{n}$ and $\mathrm{K}_{\mathrm{L}}^{0} \mathrm{p}$ channels. The overall trigger efficiency is measured to be $83 \%$. 
The mean number of $\theta^{+}$pentaquark per hadronic two-photon event extrapolated to the full phase-space for $W_{\gamma \gamma}>5 \mathrm{GeV}$ is found to be less than $4.0 \times 10^{-3}$ at $95 \%$ confidence level. The systematic uncertainty arising from the selection procedure is evaluated by varying the cuts and repeating the fitting procedure. The contribution of the $\mathrm{K}_{\mathrm{S}}^{0}$ reconstruction is estimated to be $9 \%$ whereas an uncertainty of $4 \%$ is associated with proton identification. The uncertainty due to $\theta^{+}$modelling, determined by using the $p_{t}$ and pseudo-rapidity distributions of different baryons in the $\theta^{+}$generation, is found to be $9 \%$. The effect of a non-isotropic $\theta^{+} \rightarrow \mathrm{pK}_{\mathrm{S}}^{0}$ decay on the efficiency is estimated using an angular distribution of the form $1-\cos ^{2} \alpha$, where $\alpha$ is the angle of the proton in the $\theta^{+}$centre-of-mass system. The associated systematic uncertainty is found to be $6 \%$. Limited Monte Carlo statistics introduces an additional uncertainty of $5 \%$. The sum in quadrature of these contributions yields a total systematic uncertainty of $16 \%$. Including this uncertainty in the determination of the number of $\theta^{+}$pentaquark per two-photon hadronic event gives

$$
\left\langle n_{\theta^{+}}\right\rangle<4.7 \times 10^{-3}
$$

at $95 \%$ confidence level. This result is about four times greater than the mean $\Xi^{*}(1530)$ multiplicity: a baryon with almost the same mass. This difference is mainly due to the stringent cuts applied to proton selection, resulting in a smaller selection efficiency than that of the $\Xi^{*}(1530)$ baryon, as well as the low branching ratio $\theta^{+} \rightarrow \mathrm{pK}_{\mathrm{S}}^{0}$, considered as half of that of the decay $\Xi^{*}(1530) \rightarrow \Lambda \pi^{-} \pi^{+} \rightarrow \mathrm{p} \pi^{-} \pi^{-} \pi^{+}$.

Using the two-photon cross-section for $W_{\gamma \gamma}>5 \mathrm{GeV}, \sigma_{\gamma \gamma}=397 \mathrm{nb}$ [27], a 95\% C.L. upper limit on the cross section $\gamma \gamma \rightarrow \theta^{+} X$ of $1.8 \mathrm{nb}$ is obtained. This result is comparable to the upper limit obtained by the photoproduction experiment in the reaction $\gamma \mathrm{p} \rightarrow \overline{\mathrm{K}}^{0} \theta^{+}[11]$.

As a cross check, several subsets of the selected $\mathrm{pK}_{\mathrm{S}}^{0}$ samples were investigated using tighter selection criteria. The $\mathrm{K}_{\mathrm{S}}^{0}$ selection interval was reduced to $\pm 10 \mathrm{MeV}$, the DCA of proton and antiprotons candidates was required to be less than $3 \mathrm{~mm}$ or secondary vertices compatible with the $\Lambda$ mass hypothesis were rejected. Since strangeness is a conserved quantity in strong interactions, the presence of an additional kaon was also required. No significant $\theta^{+} \operatorname{signal}$ was observed in any of these samples.

\section{Conclusion}

The production of $\Lambda, \Xi^{-}$and $\Xi^{*}(1530)$ baryons in two-photon collisions is studied in the range $0.4 \mathrm{GeV}<p_{t}<2.5 \mathrm{GeV},|\eta|<1.2$ and $W_{\gamma \gamma}>5 \mathrm{GeV}$. The shape of the differential cross section for $\Lambda$ and $\Xi^{-}$production is relatively well reproduced by the PYTHIA and PHOJET Monte Carlo programs using parameters tuned at $\sqrt{s}=m_{Z}$ although a better agreement can be obtained for $\Lambda$ by increasing the width in the gaussian $p_{t}$ distribution for primary hadrons. The mean numbers of $\Lambda, \Xi^{-}$and $\Xi^{*}(1530)$ baryons per hadronic two-photon event are found to be slightly below the PYTHIA and PHOJET predictions but overall agreement with the thermodynamical model is observed. The comparison between measurements obtained in two-photon events and $\mathrm{e}^{+} \mathrm{e}^{-}$annihilation processes provides evidence for the universality of fragmentation functions in both reactions.

Finally, a search for the pentaquark $\theta^{+}(1540)$ through the decay $\theta^{+} \rightarrow$ pK $\mathrm{S}_{\mathrm{S}}^{0}$ for $W_{\gamma \gamma}>5 \mathrm{GeV}$ is presented. No evidence for $\theta^{+}$production is found and a $95 \%$ confidence level upper limit on the mean number of $\theta^{+}$per two-photon hadronic event at a level of four times the observed rate for the $\Xi^{*}(1530)$ baryon is derived. 


\section{References}

[1] B. Andersson et al., Phys. Rep. 97 (1983) 31.

[2] B. Andersson, G. Gustafson and T. Sjöstrand, Phys. Scripta 32 (1985) 574.

[3] F. Becattini and L. Ferroni, Eur. Phys. J. C 35 (2004) 243;

F. Becattini and L. Ferroni, Eur. Phys. J. C 38 (2004) 225; and references therein.

[4] L3 Collab., P. Achard et al., Phys. Lett. B 524 (2002) 44.

[5] L3 Collab., P. Achard et al., Phys. Lett. B 586 (2004) 140.

[6] L3 Collab., B. Adeva et al., Nucl. Instr. Meth. A 289 (1990) 35;

L3 Collab., O. Adriani et al., Phys. Rept. 236 (1993) 1;

M. Chemarin et al., Nucl. Instr. Meth. A 349 (1994) 345;

M. Acciarri et al., Nucl. Instr. Meth. A 351 (1994) 300;

I. C. Brock et al., Nucl. Instr. Meth. A 381 (1996) 236;

A. Adam et al., Nucl. Instr. Meth. A 383 (1996) 342.

[7] Particle Data Group, S. Eidelman et al., Phys. Lett. B 592 (2004) 1.

[8] TOPAZ Collab., R. Enomoto et al., Phys. Lett. B 347 (1995) 179.

[9] LEPS Collab., T. Nakano et al., Phys. Rev. Lett. 91 (2003) 012002.

[10] WA89 Collab., M. I. Adamovich et al., Phys. Rev. C 72 (2005) 055201, and references therein.

[11] CLAS Collab., R. De Vita et al., hep-ex/0606062, and references therein.

[12] PYTHIA version 5.722 and JETSET version 7.409 are used with default options;

T. Sjöstrand, Comp. Phys. Comm. 82 (1994) 74.

[13] PHOJET version 1.05c is used with default options;

R. Engel, Z. Phys. C 66 (1995) 203;

R. Engel and J. Ranft, Phys. Rev. D 54 (1996) 4244.

[14] G. A. Schuler and T. Sjöstrand, Z. Phys. C 68 (1995) 607.

[15] V. M. Budnev et al., Phys. Rep. 15 (1974) 181.

[16] A. Capella et al., Phys. Rep. 236 (1994) 225.

[17] M. Glück, E. Reya and A. Vogt, Phys. Rev. D 45 (1992) 3986; Phys. Rev. D46 (1992) 1973.

[18] L3 Collab., P. Achard et al., Phys. Rept. 399 (2004) 71.

[19] KK2f version 4.12 is used;

S. Jadach, B. F. L. Ward and Z. Wạs, Comp. Phys. Comm. 130 (2000) 260.

[20] KORALZ version 4.04 is used;

S. Jadach, B. F. L. Ward and Z. Wa̧s, Comp. Phys. Comm. 79 (1994) 503. 
[21] KORALW version 1.33 is used;

M. Skrzypek et al., Comp. Phys. Comm. 94 (1996) 216.

[22] DIAG36 version 1.0 is used;

F. A. Berends, P. H. Daverfeldt and R. Kleiss, Nucl. Phys. B 253 (1985) 441.

[23] GEANT version 3.15 is used;

R. Brun et al., CERN report CERN DD/EE/84-1 (1984), revised 1987.

[24] H. Fesefeldt, RWTH Aachen report PITHA 85/2 (1985).

[25] P. Béné et al., Nucl. Inst. Meth. A 306 (1991) 150;

D. Haas et al., Nucl. Inst. Meth. A 420 (1991) 101.

[26] R. Bizzarri et al., Nucl. Instr. Meth. A 283 (1989) 799.

[27] L3 Collab., M. Acciarri et al., Phys. Lett. B 519 (2001) 33.

[28] R. D. Field and R. P. Feynman, Nucl Phys B 136 (1978) 1.

[29] F. Becattini and G. Passaleva, Eur. Phys. J. C 23 (2002) 551.

[30] P. V. Chliapnikov and V. A. Uvarov, Phys. Lett. B 345 (1995) 313. 


\section{The L3 Collaboration:}

P.Achard ${ }^{20}$ O.Adriani, ${ }^{17}$ M.Aguilar-Benitez ${ }^{25}$ J.Alcaraz $^{25}$ G.Alemanni, ${ }^{23}$ J.Allaby, ${ }^{18}$ A.Aloisio, ${ }^{29}$ M.G.Alviggi ${ }^{29}$ H.Anderhub, ${ }^{49}$ V.P.Andreev, ${ }^{64}$ F.Anselmo, A.Arefiev, ${ }^{28}$ T.Azemoon, T.Aziz, P.Bagnaia, ${ }^{39}$ A.Bajo, ${ }^{25}$ G.Baksay, ${ }^{26}$ L.Baksay, ${ }^{26}$ S.V.Baldew, ${ }^{2}$ S.Banerjee, ${ }^{9}$ Sw.Banerjee, A.Barczyk ${ }^{49,47}$ R.Barillère ${ }^{18}$ P.Bartalini2, ${ }^{23}$ M.Basile, N.Batalova, ${ }^{46}$ R.Battiston, ${ }^{33}$ A.Bay, ${ }^{23}$ F.Becattini, ${ }^{17}$ U.Becker ${ }^{13}$ F.Behner ${ }^{49}$ L.Bellucci, ${ }^{17}$ R.Berbeco, ${ }^{3}$ J.Berdugo, ${ }^{25}$ P.Berges ${ }^{13}$ B.Bertucci, ${ }^{33}$ B.L.Betev, ${ }^{49}$ M.Biasini, ${ }^{33}$ M.Biglietti ${ }^{29}$ A.Biland, ${ }^{49}$ J.J.Blaising, ${ }^{4}$ S.C.Blyth, ${ }^{35}$ G.J.Bobbink ${ }^{2}$ A.Böhm, L.Boldizsar, ${ }^{12}$ B.Borgia, ${ }^{39}$ S.Bottai, ${ }^{17}$ D.Bourilkov, ${ }^{49}$ M.Bourquin, ${ }^{20}$ S.Braccini, ${ }^{20}$ J.G.Branson, ${ }^{41}$ F.Brochu, J.D.Burger ${ }^{13}$ W.J.Burger, ${ }^{33}$ X.D.Cai ${ }^{13}$ M.Capell ${ }^{13}$ G.Cara Romeo, G.Carlino, ${ }^{29}$ A.Cartacci ${ }^{17}$ J.Casaus $^{25}$ F.Cavallari, ${ }^{39}$ N.Cavallo, ${ }^{36}$ C.Cecchi ${ }^{33}$ M.Cerrada, ${ }^{25}$ M.Chamizo, ${ }^{20}$ Y.H.Chang, ${ }^{44}$ M.Chemarin, ${ }^{24}$ A.Chen, ${ }^{44}$ G.Chen, G.M.Chen, ${ }^{7}$ H.F.Chen ${ }^{22}$ H.S.Chen, G.Chiefari, ${ }^{29}$ L.Cifarelli, ${ }^{40}$ F.Cindolo, ${ }^{8}$ I.Clare ${ }^{13}$ R.Clare, ${ }^{38}$ G.Coignet, N.Colino, ${ }^{25}$ S.Costantini, ${ }^{39}$ B.de la Cruz ${ }^{25}$ S.Cucciarelli ${ }^{33}$ R.de Asmundis, ${ }^{29}$ P.Déglon,${ }^{20}$ J.Debreczeni ${ }^{12}$ A.Degré ${ }^{4}$ K.Dehmelt ${ }^{26}$ K.Deiters ${ }^{47}$ D.della Volpe ${ }^{29}$ E.Delmeire ${ }^{20}$ P.Denes ${ }^{37}$ F.DeNotaristefani ${ }^{39}$ A.De Salvo, ${ }^{49}$ M.Diemoz ${ }^{39}$ M.Dierckxsens, ${ }^{2}$ C.Dionisi ${ }^{39}$ M.Dittmar, ${ }^{49}$ A.Doria ${ }^{29}$ M.T.Dova, ${ }^{10, \sharp}$ D.Duchesneau, ${ }^{4}$ M.Duda, ${ }^{1}$ B.Echenard, ${ }^{20}$ A.Eline ${ }^{18}$ A.El Hage, H.El Mamouni, ${ }^{14}$ A.Engler, ${ }^{35}$ F.J.Eppling, ${ }^{13}$ P.Extermann, ${ }^{20}$ M.A.Falagan, ${ }^{25}$ S.Falciano, ${ }^{39}$ A.Favara, ${ }^{32}$ J.Fay ${ }^{24}$ O.Fedin,${ }^{34}$ M.Felcini, ${ }^{49}$ T.Ferguson ${ }^{35}$ H.Fesefeldt, ${ }^{1}$ E.Fiandrini ${ }^{33}$ J.H.Field, ${ }^{20}$ F.Filthaut, ${ }^{31}$ P.H.Fisher, ${ }^{13}$ W.Fisher, ${ }^{37}$ G.Forconi ${ }^{13}$ K.Freudenreich ${ }^{49}$ C.Furetta ${ }^{27}$ Yu.Galaktionov, ${ }^{28,13}$ S.N.Ganguli, P.Garcia-Abia, ${ }^{25}$ M.Gataullin, ${ }^{32}$ S.Gentile, ${ }^{39}$ S.Giagu, ${ }^{39}$ Z.F.Gong, ${ }^{22}$ G.Grenier, ${ }^{24}$ O.Grimm, ${ }^{49}$ M.W.Gruenewald, ${ }^{16}$ V.K.Gupta, ${ }^{37}$ A.Gurtu, L.J.Gutay, ${ }^{46}$ D.Haas, D.Hatzifotiadou, ${ }^{8}$ T.Hebbeker, ${ }^{5}$ A.Hervé, ${ }^{18}$ J.Hirschfelder, ${ }^{35}$ H.Hofer, ${ }^{49}$ M.Hohlmann, ${ }^{26}$ G.Holzner, ${ }^{49}$ S.R.Hou, ${ }^{44}$ B.N.Jin, ${ }^{7}$ P.Jindal ${ }^{14}$ L.W.Jones, ${ }^{3}$ P.de Jong, ${ }^{2}$ I.Josa-Mutuberría, ${ }^{25}$ M.Kaur, ${ }^{14}$ M.N.Kienzle-Focacci, ${ }^{20}$ J.K.Kim, ${ }^{43}$ J.Kirkby, ${ }^{18}$ W.Kittel ${ }^{31}$ A.Klimentov ${ }^{13,28}$ A.C.König, ${ }^{31}$ M.Kopal, ${ }^{46}$ V.Koutsenko, ${ }^{13,28}$ M.Kräber, ${ }^{49}$ R.W.Kraemer, ${ }^{35}$ A.Krüger, ${ }^{48}$ A.Kunin, ${ }^{13}$ P.Ladron de Guevara, ${ }^{25}$ I.Laktineh, $^{24}$ G.Landi, ${ }^{17}$ M.Lebeau, ${ }^{18}$ A.Lebedev, ${ }^{13}$ P.Lebrun, ${ }^{24}$ P.Lecomte, ${ }^{49}$ P.Lecoq ${ }^{18}$ P.Le Coultre, ${ }^{49}$ J.M.Le Goff, ${ }^{18}$ R.Leiste, ${ }^{48}$ M.Levtchenko ${ }^{27}$ P.Levtchenko, ${ }^{34}$ C.Li ${ }^{22}$ S.Likhoded, ${ }^{48}$ C.H.Lin ${ }^{44}$ W.T.Lin, ${ }^{44}$ F.L.Linde, ${ }^{2}$ L.Lista, ${ }^{29}$ Z.A.Liu, W.Lohmann ${ }^{48}$ E.Longo, ${ }^{39}$ Y.S.Lu, C.Luci, ${ }^{39}$ L.Luminari ${ }^{39}$ W.Lustermann, ${ }^{49}$ W.G.Ma ${ }^{22}$ L.Malgeri ${ }^{18}$ A.Malinin, ${ }^{28}$ C.Maña, ${ }^{25}$ J.Mans, ${ }^{37}$ J.P.Martin ${ }^{24}$ F.Marzano, ${ }^{39}$ K.Mazumdar, R.R.McNeil, ${ }^{6}$ S.Mele, ${ }^{18,29}$ L.Merola, ${ }^{29}$ M.Meschini1, W.J.Metzger, ${ }^{31}$ A.Mihul, ${ }^{11}$ H.Milcent, ${ }^{18}$ G.Mirabelli, ${ }^{39}$ J.Mnich,, G.B.Mohanty, G.S.Muanza, ${ }^{24}$ A.J.M.Muijs, M.Musy, ${ }^{39}$ S.Nagy ${ }^{15}$ S.Natale, ${ }^{20}$ M.Napolitano, ${ }^{29}$ F.Nessi-Tedaldi ${ }^{49}$ H.Newman, ${ }^{32}$ A.Nisati ${ }^{39}$ T.Novak, ${ }^{31}$ H.Nowak, R.Ofierzynski, ${ }^{49}$ G.Organtini ${ }^{39}$ I.Pal ${ }^{46}$ C.Palomares, ${ }^{25}$ P.Paolucci ${ }^{29}$ R.Paramatti, ${ }^{39}$ G.Passaleva, ${ }^{17}$ S.Patricelli, ${ }^{29}$ T.Paul ${ }^{10}$ M.Pauluzzi, ${ }^{33}$ C.Paus, ${ }^{13}$ F.Pauss, ${ }^{49}$ M.Pedace, ${ }^{39}$ S.Pensotti, ${ }^{27}$ D.Perret-Gallix, ${ }^{4}$ D.Piccolo, ${ }^{29}$ F.Pierella, M.Pieri, ${ }^{41}$ M.Pioppi ${ }^{33}$ P.A.Piroué, ${ }^{37}$ E.Pistolesi ${ }^{27}$ V.Plyaskin, ${ }^{28}$ M.Pohl ${ }^{20}$ V.Pojidaev ${ }^{17}$ J.Pothier, ${ }^{18}$ D.Prokofiev ${ }^{34}$ G.Rahal-Callot, ${ }^{49}$ M.A.Rahaman, ${ }^{9}$ P.Raics ${ }^{15}$ N.Raja, ${ }^{9}$ R.Ramelli, ${ }^{49}$ P.G.Rancoita, ${ }^{27}$ R.Ranieri, ${ }^{17}$ A.Raspereza, ${ }^{48}$ P.Razis, ${ }^{30}$ S.Rembeczki, ${ }^{26}$ D.Ren, ${ }^{49}$ M.Rescigno, ${ }^{39}$ S.Reucroft ${ }^{10}$ S.Riemann, ${ }^{48}$ K.Riles, B.P.Roe, L.Romero, ${ }^{25}$ A.Rosca, ${ }^{48}$ C.Rosemann, C.Rosenbleck, ${ }^{1}$ S.Rosier-Lees, ${ }^{4}$ S.Roth, ${ }^{1}$ J.A.Rubio, ${ }^{18}$ G.Ruggiero, ${ }^{17}$ H.Rykaczewski, ${ }^{49}$ A.Sakharov, ${ }^{49}$ S.Saremi, ${ }^{6}$ S.Sarkar, ${ }^{39}$ J.Salicio, ${ }^{18}$ E.Sanchez ${ }^{25}$ C.Schäfer ${ }^{18}$ V.Schegelsky ${ }^{34}$ H.Schopper, ${ }^{21}$ D.J.Schotanus, ${ }^{31}$ C.Sciacca, ${ }^{29}$ L.Servoli ${ }^{33}$ S.Shevchenko, ${ }^{32}$ N.Shivarov, ${ }^{42}$ V.Shoutko, ${ }^{13}$ E.Shumilov, ${ }^{28}$ A.Shvorob, D.Son ${ }^{43}$ C.Souga ${ }^{24}$ P.Spillantini ${ }^{17}$ M.Steuer ${ }^{13}$ D.P.Stickland ${ }^{37}$ B.Stoyanov ${ }^{42}$ A.Straessner, ${ }^{20}$ K.Sudhakar, G.Sultanov, ${ }^{42}$ L.Z.Sun, ${ }^{22}$ S.Sushkov, H.Suter, ${ }^{49}$ J.D.Swain, ${ }^{10}$ Z.Szillasi, ${ }^{26,}$ X.W.Tang, ${ }^{7}$ P.Tarjan, ${ }^{15}$ L.Tauscher, L.Taylor, ${ }^{10}$ B.Tellili, ${ }^{24}$ D.Teyssier ${ }^{24}$ C.Timmermans, ${ }^{31}$ Samuel C.C.Ting, ${ }^{13}$ S.M.Ting, ${ }^{13}$ S.C.Tonwar, ${ }^{9}$ J.Tóth, ${ }^{12}$ C.Tully, ${ }^{37}$ K.L.Tung, J.Ulbricht, ${ }^{49}$ E.Valente, ${ }^{39}$ R.T.Van de Walle, ${ }^{31}$ R.Vasquez, ${ }^{46}$ G.Vesztergombi, ${ }^{12}$ I.Vetlitsky, ${ }^{28}$ G.Viertel ${ }^{49}$ M.Vivargent, ${ }^{4}$ S.Vlachos, ${ }^{5}$ I.Vodopianov, ${ }^{26}$ H.Vogel $^{35}{ }^{2}$ H.Vogt ${ }^{48}{ }^{2}$.Vorobiev ${ }^{35,28}$ A.A.Vorobyov, ${ }^{34}$ M.Wadhwa, ${ }^{5}$ Q.Wang ${ }^{31}$ X.L.Wang, ${ }^{22}$ Z.M.Wang, ${ }^{22}$ M.Weber ${ }^{18}{ }^{2}$ S.Wynhoff, ${ }^{37} \dagger$ L.Xia, ${ }^{32}$ Z.Z.Xu, ${ }^{22}$ J.Yamamoto, B.Z.Yang, ${ }^{22}$ C.G.Yang, H.J.Yang, ${ }^{3}$ M.Yang, S.C.Yeh, ${ }^{45}$ An.Zalite, ${ }^{34}$ Yu.Zalite, ${ }^{34}$ Z.P.Zhang, ${ }^{22}$ J.Zhao, ${ }^{22}$ G.Y.Zhu? R.Y.Zhu, ${ }^{32}$ H.L.Zhuang, A.Zichichi,, ${ }^{8,19}$ B.Zimmermann, ${ }^{49}$ M.Zöller. 
1 III. Physikalisches Institut, RWTH, D-52056 Aachen, Germany ${ }^{\S}$

2 National Institute for High Energy Physics, NIKHEF, and University of Amsterdam, NL-1009 DB Amsterdam, The Netherlands

3 University of Michigan, Ann Arbor, MI 48109, USA

4 Laboratoire d'Annecy-le-Vieux de Physique des Particules, LAPP,IN2P3-CNRS, BP 110, F-74941

Annecy-le-Vieux CEDEX, France

5 Institute of Physics, University of Basel, CH-4056 Basel, Switzerland

6 Louisiana State University, Baton Rouge, LA 70803, USA

7 Institute of High Energy Physics, IHEP, 100039 Beijing, China ${ }^{\triangle}$

8 University of Bologna and INFN-Sezione di Bologna, I-40126 Bologna, Italy

9 Tata Institute of Fundamental Research, Mumbai (Bombay) 400 005, India

10 Northeastern University, Boston, MA 02115, USA

11 Institute of Atomic Physics and University of Bucharest, R-76900 Bucharest, Romania

12 Central Research Institute for Physics of the Hungarian Academy of Sciences, H-1525 Budapest 114, Hungary ${ }^{\ddagger}$

13 Massachusetts Institute of Technology, Cambridge, MA 02139, USA

14 Panjab University, Chandigarh 160 014, India

15 KLTE-ATOMKI, H-4010 Debrecen, Hungary ${ }^{\circledR}$

16 UCD School of Physics, University College Dublin, Belfield, Dublin 4, Ireland

17 INFN Sezione di Firenze and University of Florence, I-50125 Florence, Italy

18 European Laboratory for Particle Physics, CERN, CH-1211 Geneva 23, Switzerland

19 World Laboratory, FBLJA Project, CH-1211 Geneva 23, Switzerland

20 University of Geneva, CH-1211 Geneva 4, Switzerland

21 University of Hamburg, D-22761 Hamburg, Germany

22 Chinese University of Science and Technology, USTC, Hefei, Anhui 230 029, China ${ }^{\triangle}$

23 University of Lausanne, CH-1015 Lausanne, Switzerland

24 Institut de Physique Nucléaire de Lyon, IN2P3-CNRS,Université Claude Bernard, F-69622 Villeurbanne, France

25 Centro de Investigaciones Energéticas, Medioambientales y Tecnológicas, CIEMAT, E-28040 Madrid, Spainb

26 Florida Institute of Technology, Melbourne, FL 32901, USA

27 INFN-Sezione di Milano, I-20133 Milan, Italy

28 Institute of Theoretical and Experimental Physics, ITEP, Moscow, Russia

29 INFN-Sezione di Napoli and University of Naples, I-80125 Naples, Italy

30 Department of Physics, University of Cyprus, Nicosia, Cyprus

31 Radboud University and NIKHEF, NL-6525 ED Nijmegen, The Netherlands

32 California Institute of Technology, Pasadena, CA 91125, USA

33 INFN-Sezione di Perugia and Università Degli Studi di Perugia, I-06100 Perugia, Italy

34 Nuclear Physics Institute, St. Petersburg, Russia

35 Carnegie Mellon University, Pittsburgh, PA 15213, USA

36 INFN-Sezione di Napoli and University of Potenza, I-85100 Potenza, Italy

37 Princeton University, Princeton, NJ 08544, USA

38 University of Californa, Riverside, CA 92521, USA

39 INFN-Sezione di Roma and University of Rome, "La Sapienza", I-00185 Rome, Italy

40 University and INFN, Salerno, I-84100 Salerno, Italy

41 University of California, San Diego, CA 92093, USA

42 Bulgarian Academy of Sciences, Central Lab. of Mechatronics and Instrumentation, BU-1113 Sofia, Bulgaria

43 The Center for High Energy Physics, Kyungpook National University, 702-701 Taegu, Republic of Korea

44 National Central University, Chung-Li, Taiwan, China

45 Department of Physics, National Tsing Hua University, Taiwan, China

46 Purdue University, West Lafayette, IN 47907, USA

47 Paul Scherrer Institut, PSI, CH-5232 Villigen, Switzerland

48 DESY, D-15738 Zeuthen, Germany

49 Eidgenössische Technische Hochschule, ETH Zürich, CH-8093 Zürich, Switzerland

$\S$ Supported by the German Bundesministerium für Bildung, Wissenschaft, Forschung und Technologie.

¥ Supported by the Hungarian OTKA fund under contract numbers T019181, F023259 and T037350.

ฯ Also supported by the Hungarian OTKA fund under contract number T026178.

b Supported also by the Comisión Interministerial de Ciencia y Tecnología.

\# Also supported by CONICET and Universidad Nacional de La Plata, CC 67, 1900 La Plata, Argentina.

$\triangle$ Supported by the National Natural Science Foundation of China.

$\dagger$ Deceased. 


\begin{tabular}{|c|c|c|c|c|c|c|}
\hline $\begin{array}{c}p_{t} \\
(\mathrm{GeV})\end{array}$ & $\begin{array}{c}\left\langle p_{t}\right\rangle \\
(\mathrm{GeV})\end{array}$ & $\begin{array}{c}\left\langle p_{t}^{2}\right\rangle \\
\left(\mathrm{GeV}^{2}\right)\end{array}$ & $\begin{array}{c}\text { Number of } \\
\text { baryons }\end{array}$ & $\begin{array}{c}\text { Efficiency } \\
(\%)\end{array}$ & $\begin{array}{c}\mathrm{d} \sigma / \mathrm{d} p_{t} \\
(\mathrm{pb} / \mathrm{GeV})\end{array}$ & $\begin{array}{c}\mathrm{d} \sigma / \mathrm{d} p_{t}^{2} \\
\left(\mathrm{pb} / \mathrm{GeV}^{2}\right)\end{array}$ \\
\hline \multicolumn{7}{|c|}{$\Lambda$ baryon } \\
\hline $0.4-0.6$ & 0.50 & 0.25 & $3412 \pm 71$ & $10.2 \pm 0.1$ & $273.1 \pm 5.7 \pm 35.7$ & $273.1 \pm 5.7 \pm 35.7$ \\
\hline $0.6-0.8$ & 0.69 & 0.48 & $4408 \pm 85$ & $13.7 \pm 0.1$ & $264.1 \pm 5.1 \pm 29.1$ & $188.7 \pm 3.6 \pm 20.8$ \\
\hline $0.8-1.0$ & 0.89 & 0.79 & $3420 \pm 81$ & $15.3 \pm 0.2$ & $183.3 \pm 4.4 \pm 15.3$ & $101.9 \pm 2.4 \pm 8.5$ \\
\hline $1.0-1.3$ & 1.12 & 1.27 & $3201 \pm 87$ & $16.8 \pm 0.2$ & $103.9 \pm 2.8 \pm 7.8$ & $45.2 \pm 1.2 \pm 3.4$ \\
\hline $1.3-1.6$ & 1.43 & 2.05 & $1222 \pm 55$ & $17.7 \pm 0.4$ & $37.8 \pm 1.7 \pm$ & $13.0 \pm 0.6 \pm$ \\
\hline $1.6-2.0$ & 1.77 & 3.14 & $578 \pm 41$ & $15.9 \pm 0.6$ & $15.0 \pm 1.1 \pm$ & $4.2 \pm 0.3 \pm 0.4$ \\
\hline $2.0-2.5$ & 2.21 & 4.92 & $292 \pm 25$ & $17.2 \pm 1.3$ & $5.6 \pm 0.5 \pm$ & $1.2 \pm 0.1 \pm$ \\
\hline \multicolumn{7}{|c|}{$\Xi^{-}$baryon } \\
\hline $0.4-0.7$ & 0.55 & 0.31 & $70 \pm 10$ & $3.4 \pm 0.2$ & $11.3 \pm 1.7 \pm$ & $10.3 \pm 1.5 \pm 1.6$ \\
\hline $0.7-1.0$ & 0.83 & 0.70 & $113 \pm 12$ & $7.1 \pm 0.3$ & $8.7 \pm 1.2 \pm$ & $5.1 \pm 0.6 \pm 0.7$ \\
\hline $1.0-1.3$ & 1.13 & 1.27 & $83 \pm 12$ & $9.7 \pm 0.8$ & $4.7 \pm 0.8 \pm$ & $2.0 \pm 0.3 \pm$ \\
\hline $1.3-2.5$ & 1.67 & 2.88 & $94 \pm 19$ & $8.8 \pm 0.9$ & $1.5 \pm 0.3 \pm$ & $0.4 \pm 0.1 \pm$ \\
\hline \multicolumn{7}{|c|}{$\Xi^{*}(1530)$ baryon } \\
\hline $0.4-2.5$ & 0.82 & 0.84 & $56 \pm 13$ & $3.8 \pm 0.6$ & $1.4 \pm 0.3 \pm 0.5$ & $0.5 \pm 0.1 \pm 0.2$ \\
\hline
\end{tabular}

Table 1: The average transverse momentum $\left\langle p_{t}\right\rangle$, the average transverse momentum squared $\left\langle p_{t}^{2}\right\rangle$, the number of baryons estimated by the fits to the $\Lambda, \Xi^{-}$and $\Xi^{*}(1530)$ mass spectra, the detection efficiency and the differential cross sections $\mathrm{d} \sigma / \mathrm{d} p_{t}$ and $\mathrm{d} \sigma / \mathrm{d} p_{t}^{2}$ for inclusive $\Lambda, \Xi^{-}$ and $\Xi^{*}(1530)$ production as a function of $p_{t}$ for $|\eta|<1.2$. The first uncertainty on the cross sections is statistical and the second systematic.

\begin{tabular}{|c|c|c|c|c|}
\hline$|\eta|$ & $\langle|\eta|\rangle$ & $\begin{array}{c}\text { Number of } \\
\text { baryons }\end{array}$ & $\begin{array}{c}\text { Efficiency } \\
(\%)\end{array}$ & $\begin{array}{c}\mathrm{d} \sigma / \mathrm{d}|\eta| \\
(\mathrm{pb})\end{array}$ \\
\hline \multicolumn{5}{|c|}{$\Lambda$ baryon $0.4 \mathrm{GeV}<p_{t}<1.0 \mathrm{GeV}$} \\
\hline $0.0-0.3$ & 0.15 & $2953 \pm 72$ & $13.8 \pm 0.2$ & $58.6 \pm 1.4 \pm 3.4$ \\
$0.3-0.6$ & 0.45 & $2742 \pm 63$ & $13.4 \pm 0.2$ & $56.1 \pm 1.3 \pm 3.2$ \\
$0.6-0.9$ & 0.75 & $2904 \pm 70$ & $13.0 \pm 0.2$ & $61.0 \pm 1.5 \pm 4.1$ \\
$0.9-1.2$ & 1.05 & $2774 \pm 89$ & $11.1 \pm 0.1$ & $68.0 \pm 2.2 \pm 8.4$ \\
\hline \multicolumn{5}{|c|}{$\Lambda$ baryon $1.0 \mathrm{GeV}<p_{t}<2.5 \mathrm{GeV}$} \\
\hline $0.0-0.3$ & 0.15 & $1458 \pm 60$ & $18.8 \pm 0.5$ & $21.2 \pm 0.9 \pm 1.6$ \\
$0.3-0.6$ & 0.45 & $1411 \pm 62$ & $18.2 \pm 0.4$ & $21.2 \pm 0.9 \pm 1.6$ \\
$0.6-0.9$ & 0.75 & $1480 \pm 62$ & $19.3 \pm 0.5$ & $20.9 \pm 0.9 \pm 1.6$ \\
$0.9-1.2$ & 1.05 & $1007 \pm 62$ & $14.3 \pm 0.4$ & $19.3 \pm 1.2 \pm 2.7$ \\
\hline \multicolumn{5}{|}{- baryon $0.4 \mathrm{GeV}<p_{t}<2.5 \mathrm{GeV}$} \\
\hline $0.0-0.4$ & 0.20 & $110 \pm 14$ & $6.7 \pm 0.3$ & $6.9 \pm 0.9 \pm 1.0$ \\
$0.4-0.8$ & 0.60 & $114 \pm 13$ & $6.1 \pm 0.2$ & $8.0 \pm 1.0 \pm 1.0$ \\
$0.8-1.2$ & 1.01 & $109 \pm 15$ & $5.5 \pm 0.2$ & $7.6 \pm 1.1 \pm 1.2$ \\
\hline
\end{tabular}

Table 2: The average pseudo-rapidity $\langle|\eta|\rangle$, the number of baryons estimated by the fits to the $\Lambda$ and $\Xi^{-}$mass spectra, the detection efficiency and the differential cross sections $\mathrm{d} \sigma / \mathrm{d}|\eta|$ for inclusive $\Lambda$ and $\Xi^{-}$production as a function of $|\eta|$. The first uncertainty on the cross sections is statistical and the second systematic. 


\begin{tabular}{|l|c|c|c|}
\cline { 2 - 4 } \multicolumn{1}{c|}{} & Data & \multicolumn{1}{c|}{ PYTHIA } & PHOJET \\
\cline { 2 - 4 } \multicolumn{1}{c|}{} & \multicolumn{2}{c|}{$0.4 \mathrm{GeV}<p_{t}<2.5 \mathrm{GeV},|\eta|<1.2$ and $W_{\gamma \gamma}>5 \mathrm{GeV}$} \\
\hline$\left\langle n_{\Lambda}\right\rangle$ & $(1.6 \pm 0.1) \times 10^{-2}$ & $(1.43 \pm 0.01) \times 10^{-2}$ & $(1.80 \pm 0.01) \times 10^{-2}$ \\
$\left\langle n_{\Xi^{-}}\right\rangle$ & $(7.6 \pm 1.0) \times 10^{-4}$ & $(8.61 \pm 0.11) \times 10^{-4}$ & $(11.5 \pm 0.10) \times 10^{-4}$ \\
$\left\langle n_{\Xi^{*}(1530)}\right\rangle$ & $(2.3 \pm 1.0) \times 10^{-4}$ & $(1.37 \pm 0.04) \times 10^{-4}$ & $(1.91 \pm 0.03) \times 10^{-4}$ \\
\hline
\end{tabular}

\begin{tabular}{|l|c|c|c|}
\cline { 2 - 4 } \multicolumn{1}{c|}{} & Data & PYTHIA & PHOJET \\
\cline { 2 - 4 } \multicolumn{1}{c|}{} & \multicolumn{3}{|c|}{$W_{\gamma \gamma}>5 \mathrm{GeV}$} \\
\hline$\left\langle n_{\Lambda}\right\rangle$ & $(7.6 \pm 0.8) \times 10^{-2}$ & $(8.51 \pm 0.01) \times 10^{-2}$ & $(1.11 \pm 0.01) \times 10^{-1}$ \\
$\left\langle n_{\Xi^{-}}\right\rangle$ & $(3.7 \pm 0.5) \times 10^{-3}$ & $(5.17 \pm 0.02) \times 10^{-3}$ & $(7.29 \pm 0.09) \times 10^{-3}$ \\
$\left\langle n_{\Xi^{*}(1530)}\right\rangle$ & $(11.8 \pm 5.2) \times 10^{-4}$ & $(8.65 \pm 0.09) \times 10^{-4}$ & $(12.4 \pm 0.10) \times 10^{-3}$ \\
\hline
\end{tabular}

Table 3: The mean number of $\Lambda, \Xi^{-}$and $\Xi^{*}(1530)$ baryons per hadronic two-photon event for the measured $p_{t}$ and $|\eta|$ phase-space (top) and its extrapolation (bottom) to the full $p_{t}$ and $|\eta|$ range. The uncertainty on data includes both the statistical and systematic contributions whereas the uncertainty on the Monte Carlo predictions is statistical.

\begin{tabular}{|c|c|c|c|c|r|}
\hline $\begin{array}{c}p_{t} \\
(\mathrm{GeV})\end{array}$ & $\begin{array}{c}\text { Selection } \\
\text { criteria }\end{array}$ & $\begin{array}{c}\text { Background } \\
\text { subtraction }\end{array}$ & $\begin{array}{c}\text { Monte Carlo } \\
\text { statistics }\end{array}$ & $\begin{array}{c}\text { Monte Carlo } \\
\text { modelling }\end{array}$ & Total \\
\hline \multicolumn{6}{|c|}{$\Lambda$ baryon } \\
\hline $0.4-0.6$ & 4.2 & 12.1 & 0.9 & 1.4 & 13.1 \\
$0.6-0.8$ & 4.2 & 9.8 & 0.9 & 1.6 & 11.0 \\
$0.8-1.0$ & 4.2 & 6.6 & 1.2 & 1.9 & 8.4 \\
$1.0-1.3$ & 4.2 & 5.3 & 1.4 & 2.1 & 7.5 \\
$1.3-1.6$ & 4.2 & 5.8 & 2.4 & 2.4 & 8.2 \\
$1.6-2.0$ & 4.2 & 6.4 & 3.5 & 2.8 & 9.1 \\
$2.0-2.5$ & 4.2 & 19.4 & 7.5 & 4.8 & 21.8 \\
\hline \multicolumn{6}{|c|}{$\Xi$ baryon } \\
\hline $0.4-0.7$ & 10.3 & 8.7 & 5.8 & 3.1 & 15.1 \\
$0.7-1.0$ & 10.3 & 7.5 & 4.8 & 2.4 & 14.0 \\
$1.0-1.3$ & 10.3 & 9.1 & 8.3 & 3.3 & 16.5 \\
$1.3-2.5$ & 10.3 & 12.3 & 10.4 & 6.3 & 19.5 \\
\hline \multicolumn{7}{|c|}{$\Xi^{*}(1530)$ baryon } \\
\hline $0.4-2.5$ & 19.2 & 24.6 & 16.5 & 13.6 & 37.9 \\
\hline
\end{tabular}

Table 4: Systematic uncertainties in percent on the cross section of the $\mathrm{e}^{+} \mathrm{e}^{-} \rightarrow \mathrm{e}^{+} \mathrm{e}^{-} \Lambda \mathrm{X}$, $\mathrm{e}^{+} \mathrm{e}^{-} \rightarrow \mathrm{e}^{+} \mathrm{e}^{-} \Xi^{-} \mathrm{X}$ and $\mathrm{e}^{+} \mathrm{e}^{-} \rightarrow \mathrm{e}^{+} \mathrm{e}^{-} \Xi^{*}(1530) \mathrm{X}$ processes due to selection criteria, background subtraction, limited Monte Carlo statistics, Monte Carlo modelling and trigger efficiency as a function of $p_{t}$ for $|\eta|<1.2$. The total systematic uncertainty, taken as the quadratic sum of the different contributions, includes a constant contribution of $2 \%$ due to the determination of the trigger efficiency. 

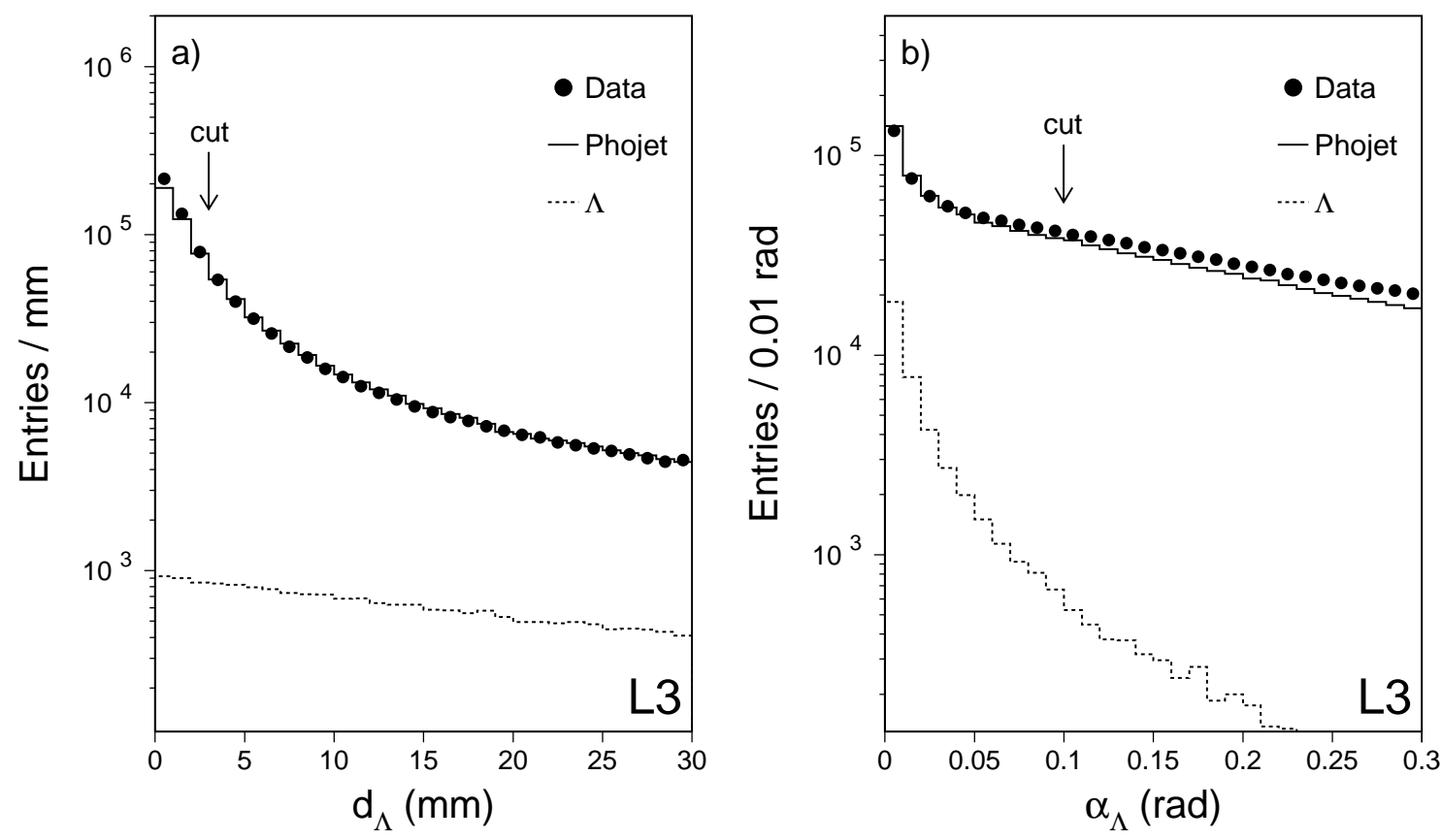

Figure 1: Distribution of the variables used to select secondary vertices from inclusive $\Lambda$ production: a) the distance in the transverse plane between the secondary vertex and the $\mathrm{e}^{+} \mathrm{e}^{-}$ interaction point, $\mathrm{d}_{\Lambda}$, and $\mathrm{b}$ ) the angle between the sum $p_{t}$ vector of the two tracks and the direction in the transverse plane between the interaction point and the secondary vertex, $\alpha_{\Lambda}$. All other secondary vertex selection criteria are applied. The predictions of the PHOJET Monte Carlo are shown as the solid lines and the contribution due to $\Lambda$ baryons as the dashed lines. The Monte Carlo distributions are normalized to the data luminosity. 

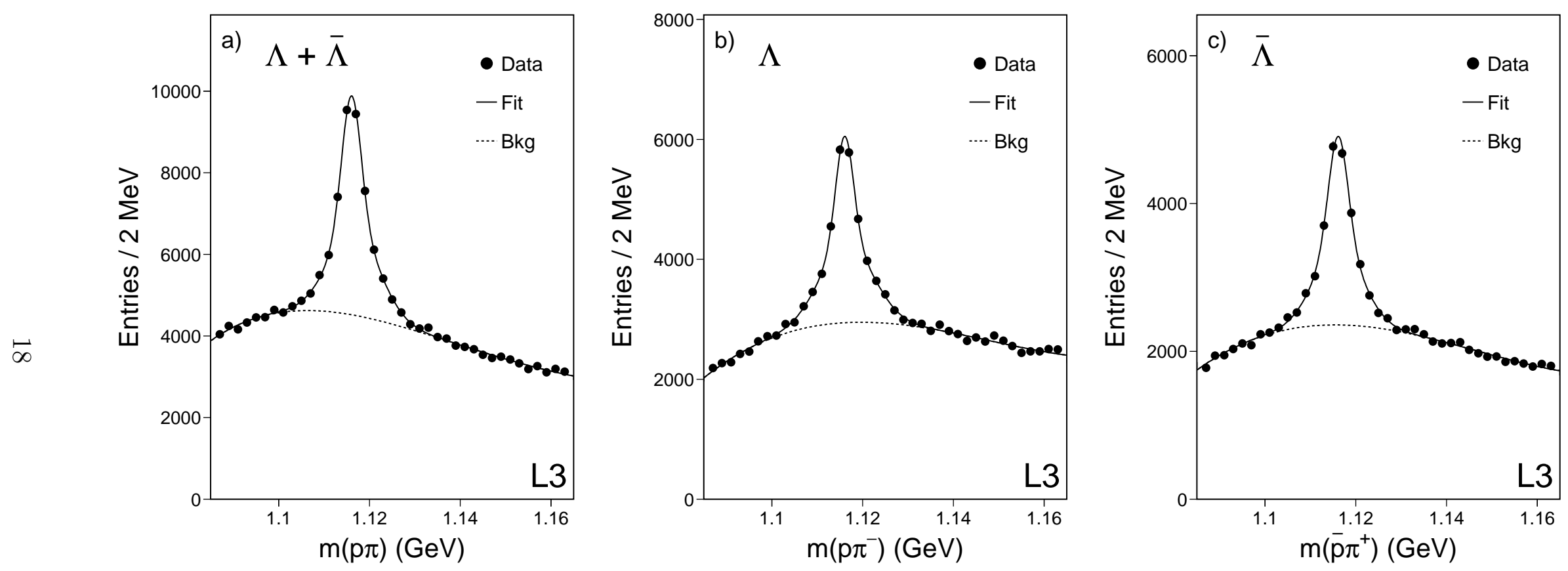

Figure 2: The mass of the p $\pi$ system for the inclusive $\Lambda$ selection for a) $\Lambda$ and $\bar{\Lambda}$ candidates, b) $\Lambda$ candidates and c) $\bar{\Lambda}$ candidates. The signal is modelled with two Gaussians and the background by a fourth-order Chebyshev polynomial. 

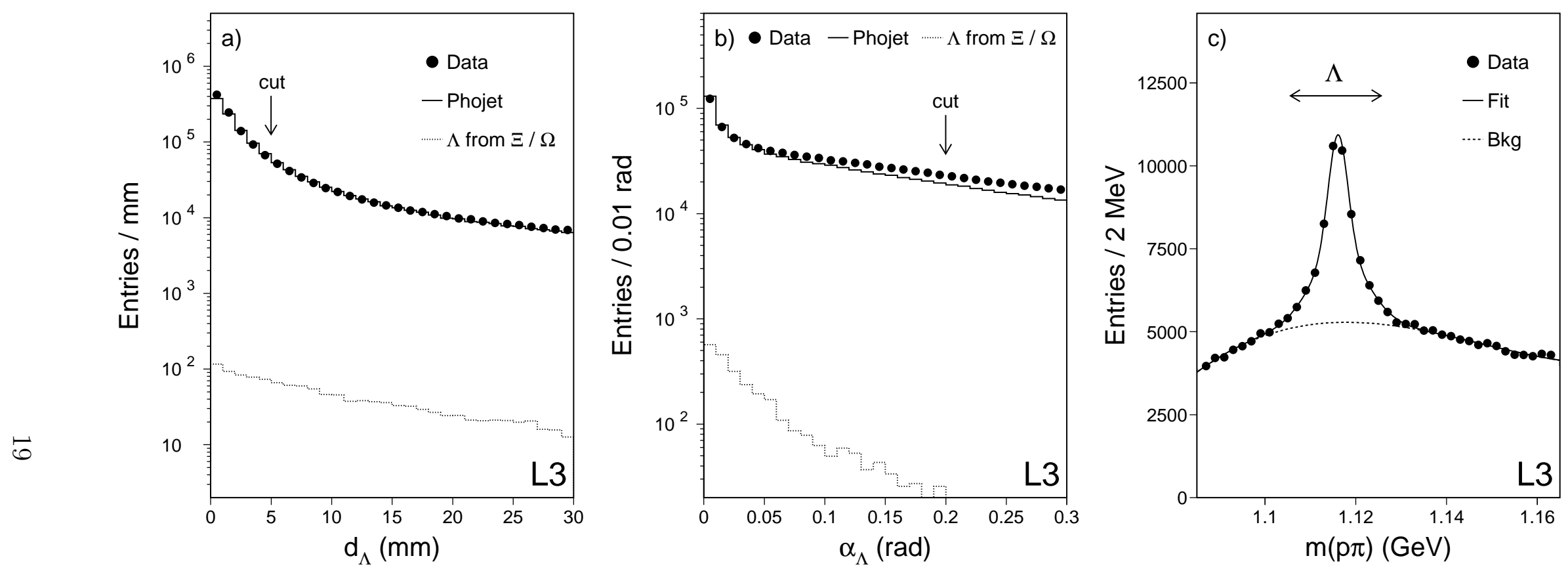

Figure 3: Distribution of the variables used to select secondary vertices from $\Lambda$ produced in $\Xi^{-}$and $\Omega^{-}$decays: a) the distance in the transverse plane between the secondary vertex and the $\mathrm{e}^{+} \mathrm{e}^{-}$interaction point, $\mathrm{d}_{\Lambda}$, and $\mathrm{b}$ ) the angle between the sum $p_{t}$ vector of the two tracks and the direction in the transverse plane between the interaction point and the secondary vertex, $\alpha_{\Lambda}$. All other secondary vertex selection criteria are applied. The predictions of the PHOJET Monte Carlo are shown as the full line and the contribution due to $\Lambda$ baryons as the dashed line. c) The mass of the $\mathrm{p} \pi$ system for $\Lambda$ baryons produced in $\Xi^{-}$and $\Omega^{-}$decays. The signal is modelled with two Gaussians and the background by a fourth-order Chebyshev polynomial. Only the combinations in the $\pm 10 \mathrm{MeV}$ mass window indicated by the arrows are retained to reconstruct $\Xi^{-}$and $\Omega^{-}$baryons. The Monte Carlo distributions are normalized to the data luminosity. 

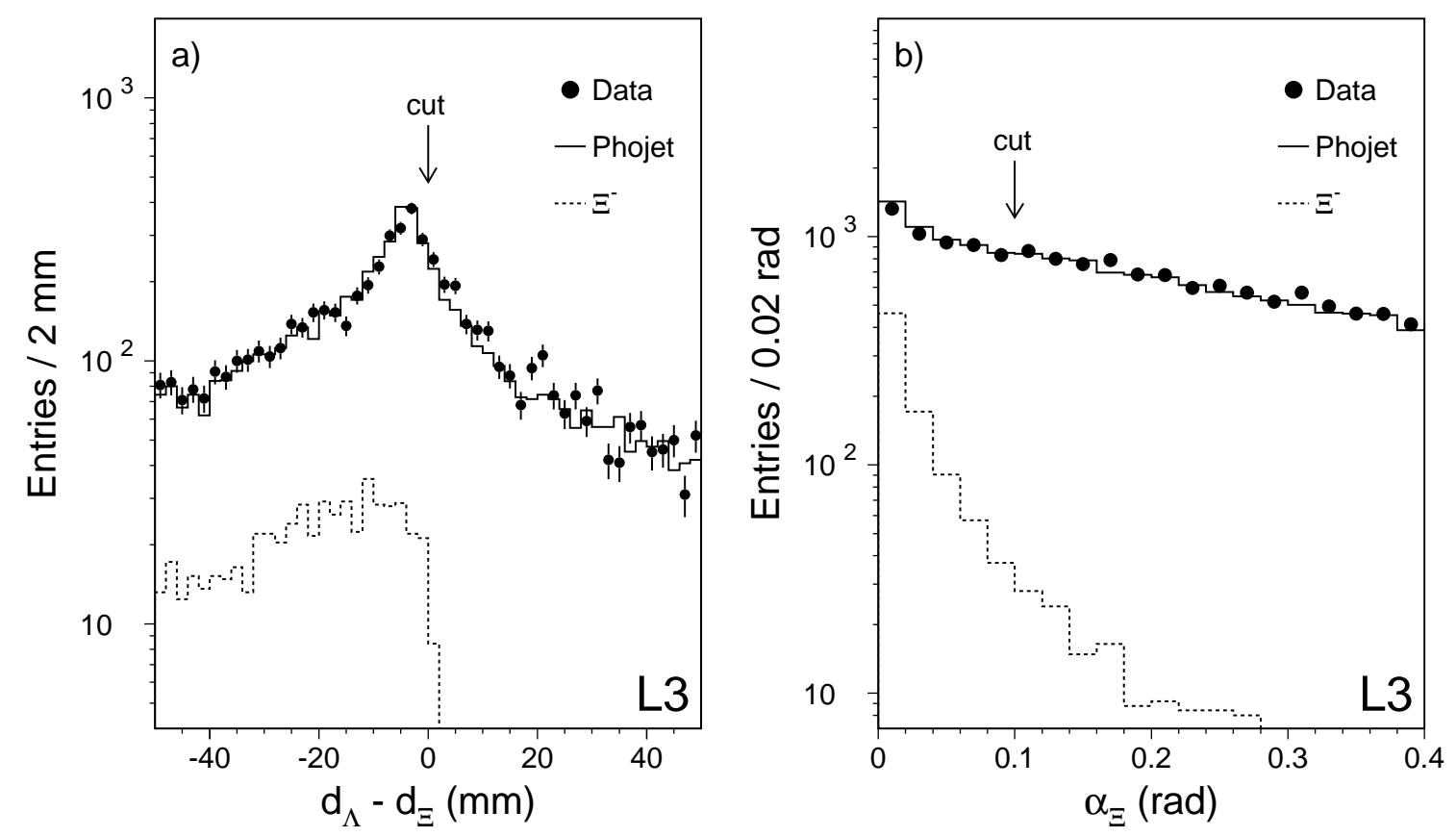

Figure 4: Distribution of the variables used for the selection of $\Xi^{-}$baryons: a) the difference $d_{\Lambda}-d_{\Xi}$ of the $\Lambda$ and $\Xi^{-}$vertex distances from the interaction point and b) the angle $\alpha_{\Xi}$ between the $p_{t}$ vector of the $\Lambda \pi$ combination and the direction in the transverse plane between the primary interaction point and the $\Lambda \pi$ vertex. In each plot, all other selection criteria are applied. The predictions of the PHOJET Monte Carlo are shown as the full line and the contribution due to $\Xi^{-}$baryons as the dashed line. The Monte Carlo distributions are normalized to the data luminosity. 

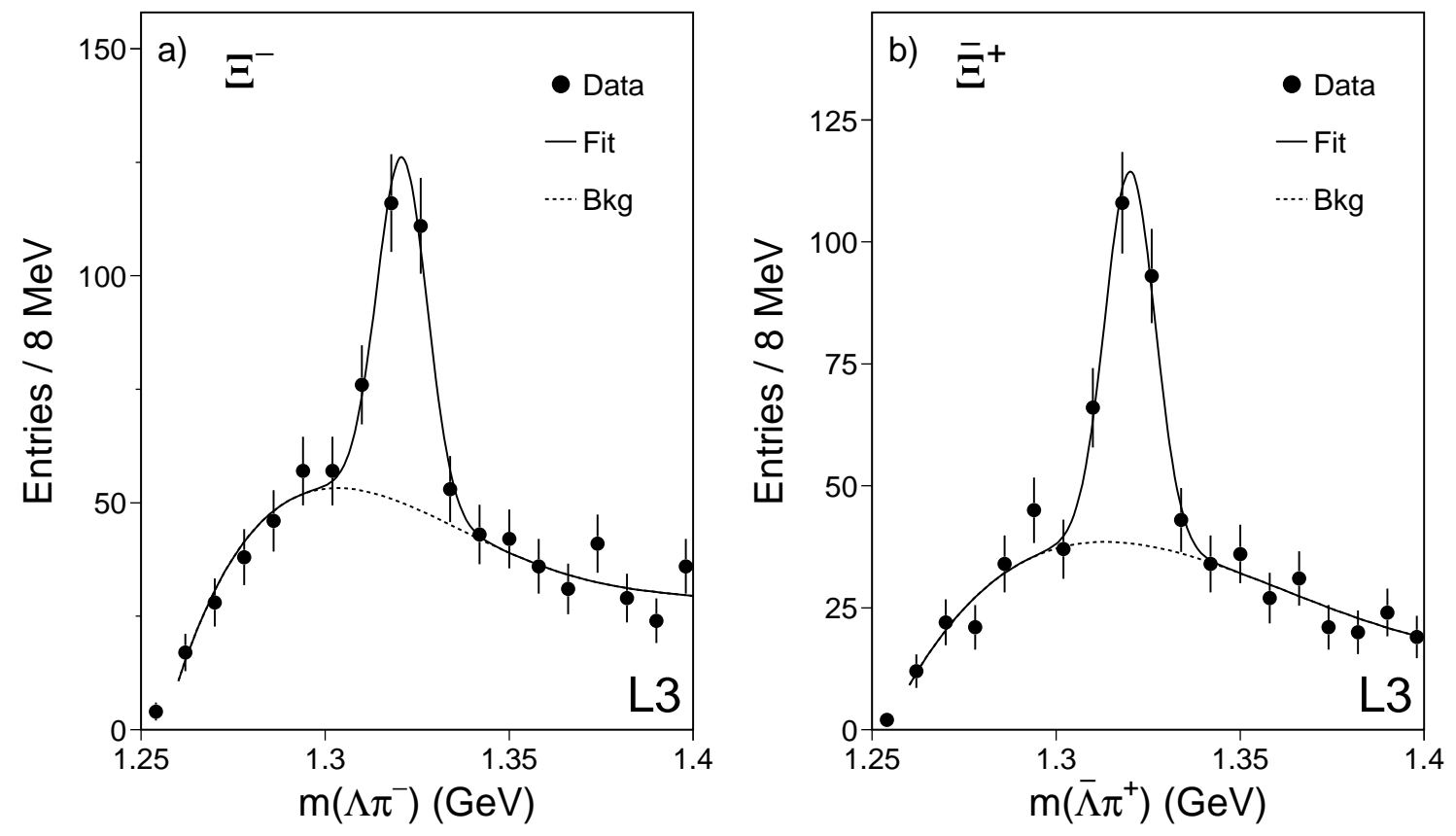

Figure 5: The mass spectrum of the $\Lambda \pi$ system for a) $\Xi^{-}$and b) $\bar{\Xi}^{+}$candidates for $0.4 \mathrm{GeV}<$ $p_{t}<2.5 \mathrm{GeV}$ and $|\eta|<1.2$. The signal is modelled with a Gaussian and the background by a fourth order Chebyshev polynomial. 

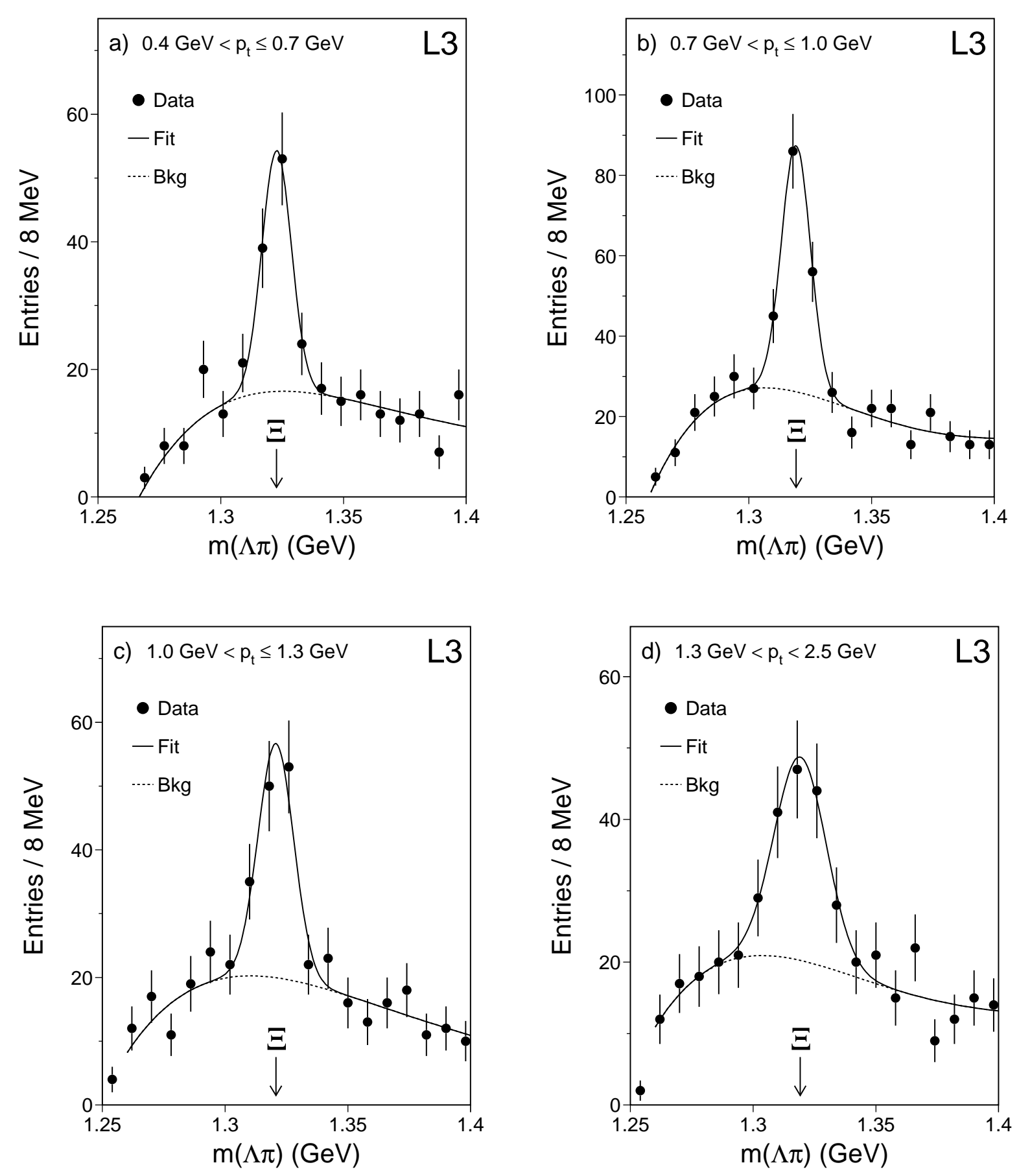

Figure 6: The mass of the $\Lambda \pi$ system for a) $0.4 \mathrm{GeV}<p_{t} \leq 0.7 \mathrm{GeV}$, b) $0.7 \mathrm{GeV}<p_{t} \leq$ $1.0 \mathrm{GeV}$, c) $1.0 \mathrm{GeV}<p_{t}<1.3 \mathrm{GeV}$ and d) $1.3 \mathrm{GeV}<p_{t}<2.5 \mathrm{GeV}$. The signal is modelled with a Gaussian and the background by a fourth-order Chebyshev polynomial. 

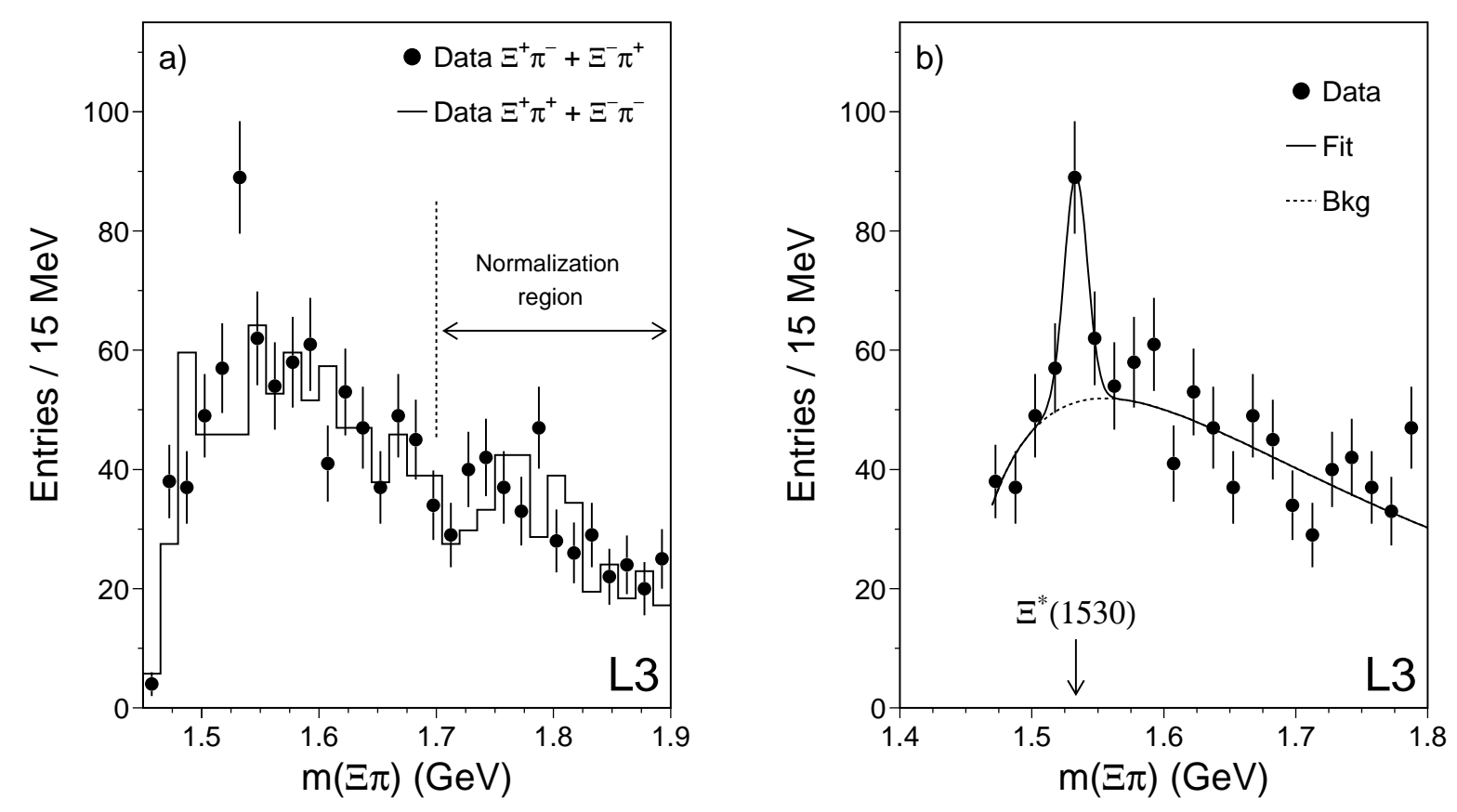

Figure 7: a) The mass of the $\Xi \pi$ system for opposite charge $\left(\Xi^{-} \pi^{+}\right.$and $\left.\bar{\Xi}^{+} \pi^{-}\right)$and same-charge $\left(\Xi^{-} \pi^{-}\right.$and $\left.\bar{\Xi}^{+} \pi^{+}\right)$combinations. The number of same-charge combinations is normalized to that of opposite-charge combinations in the region $m_{\Xi \pi}>1.7 \mathrm{GeV}$. A signal consistent with $\Xi^{*}(1530)$ production is observed. b) Fit to the mass spectrum of the opposite-charge combinations. The signal is modelled with a Gaussian and the background by a threshold function. 


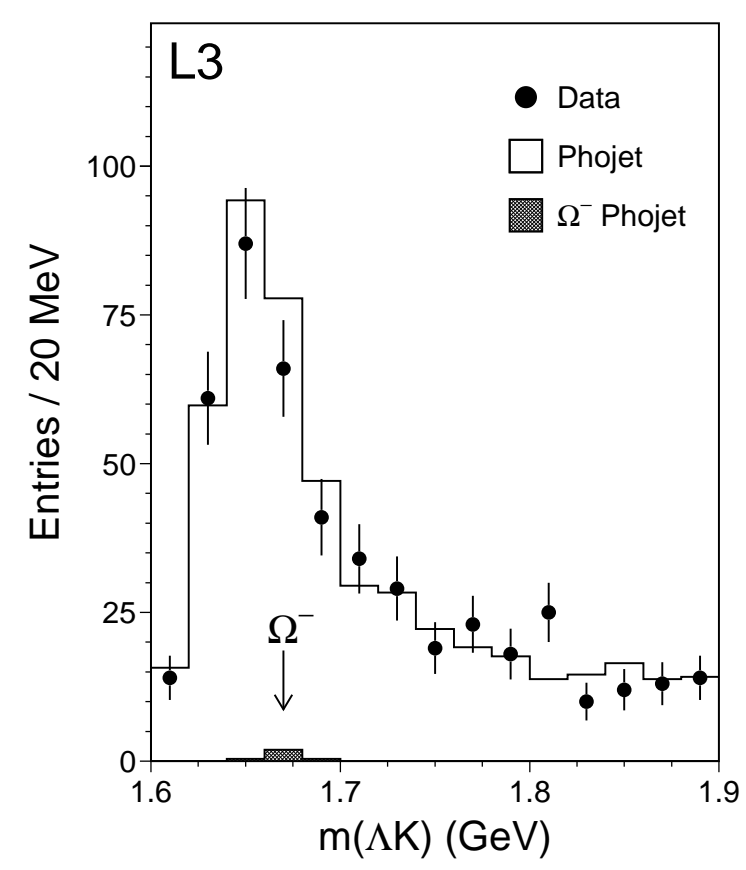

Figure 8: The mass of the $\Lambda \mathrm{K}$ system. No $\Omega^{-}$signal is observed around the mass of $1.67 \mathrm{GeV}$. The predictions of the PHOJET Monte Carlo are shown as the full line and the contribution due to $\Omega^{-}$baryons as the dashed histogram. The Monte Carlo distributions are normalized to the data luminosity.
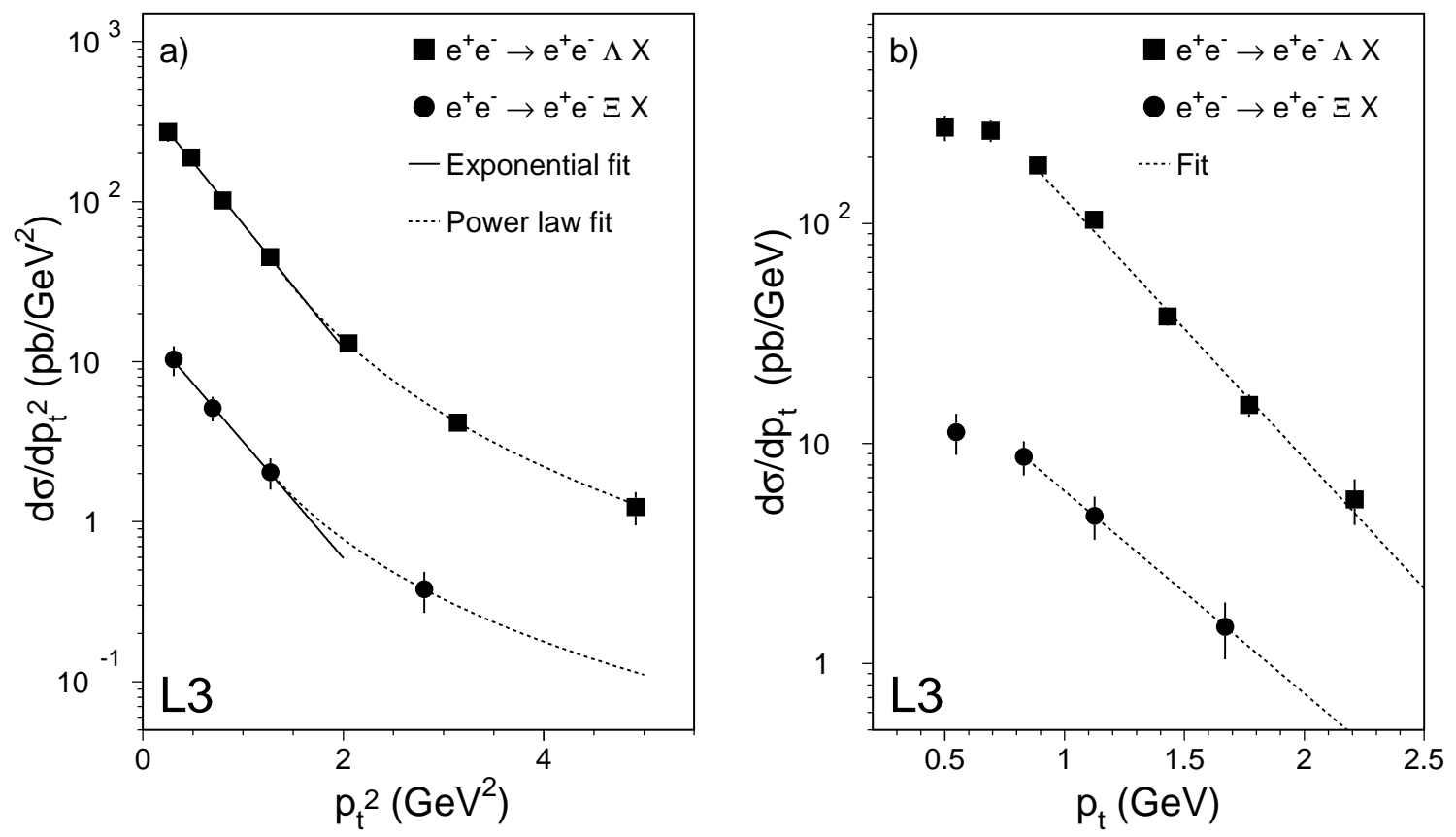

Figure 9: a) Differential cross sections $\mathrm{d} \sigma / \mathrm{d} p_{t}^{2}$ for the $\mathrm{e}^{+} \mathrm{e}^{-} \rightarrow \mathrm{e}^{+} \mathrm{e}^{-} \Lambda \mathrm{X}$ and $\mathrm{e}^{+} \mathrm{e}^{-} \rightarrow \mathrm{e}^{+} \mathrm{e}^{-} \Xi^{-} \mathrm{X}$ processes for $|\eta|<1.2$. b) Differential cross sections $\mathrm{d} \sigma / \mathrm{d} p_{t}$ for the $\mathrm{e}^{+} \mathrm{e}^{-} \rightarrow \mathrm{e}^{+} \mathrm{e}^{-} \Lambda \mathrm{X}$ and $\mathrm{e}^{+} \mathrm{e}^{-} \rightarrow \mathrm{e}^{+} \mathrm{e}^{-} \Xi^{-} \mathrm{X}$ processes for $|\eta|<1.2$. The various fits are described in the text. 

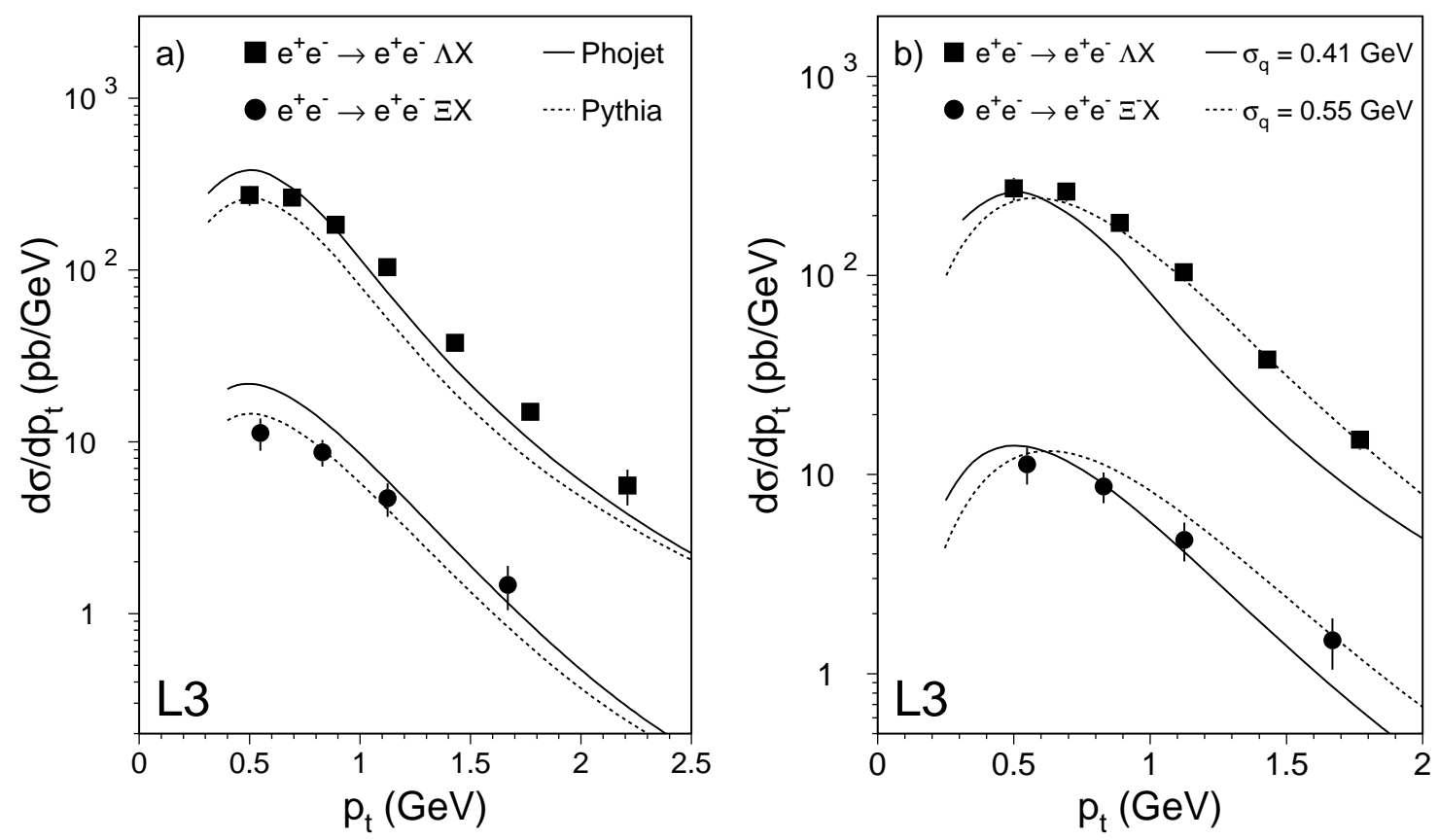

Figure 10: The differential cross section as a function of $p_{t}$ for $\Lambda$ and $\Xi^{-}$production for $|\eta|<1.2$ compared with a) the predictions of the PHOJET and PYTHIA Monte Carlo, and b) the predictions of the PYTHIA Monte Carlo using the default value for the width $\sigma_{q}$ in the Gaussian $p_{t}$ distribution on primary hadrons $\sigma_{q}=0.411 \mathrm{GeV}$ as well as the adjusted value $\sigma_{q}=0.55 \mathrm{GeV}$. 

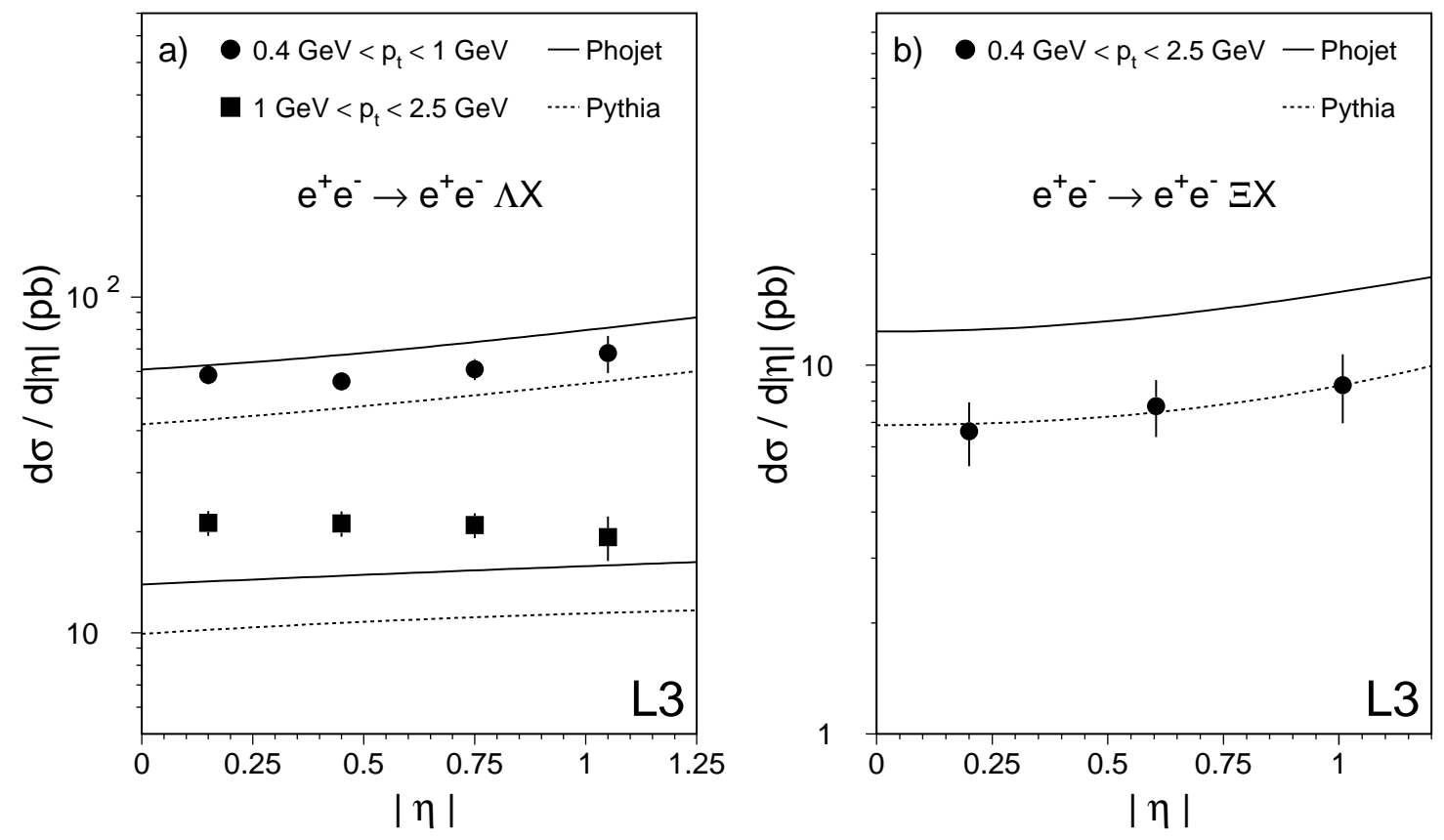

Figure 11: The differential cross section as a function of $|\eta|$ for a) inclusive $\Lambda$ production for $0.4 \mathrm{GeV}<p_{t}<1.0 \mathrm{GeV}$ and $1.0 \mathrm{GeV}<p_{t}<2.5 \mathrm{GeV}$ and b) for inclusive $\Xi^{-}$production for $0.4 \mathrm{GeV}<p_{t}<2.5 \mathrm{GeV}$. The predictions of the PHOJET and PYTHIA programs are also shown. 


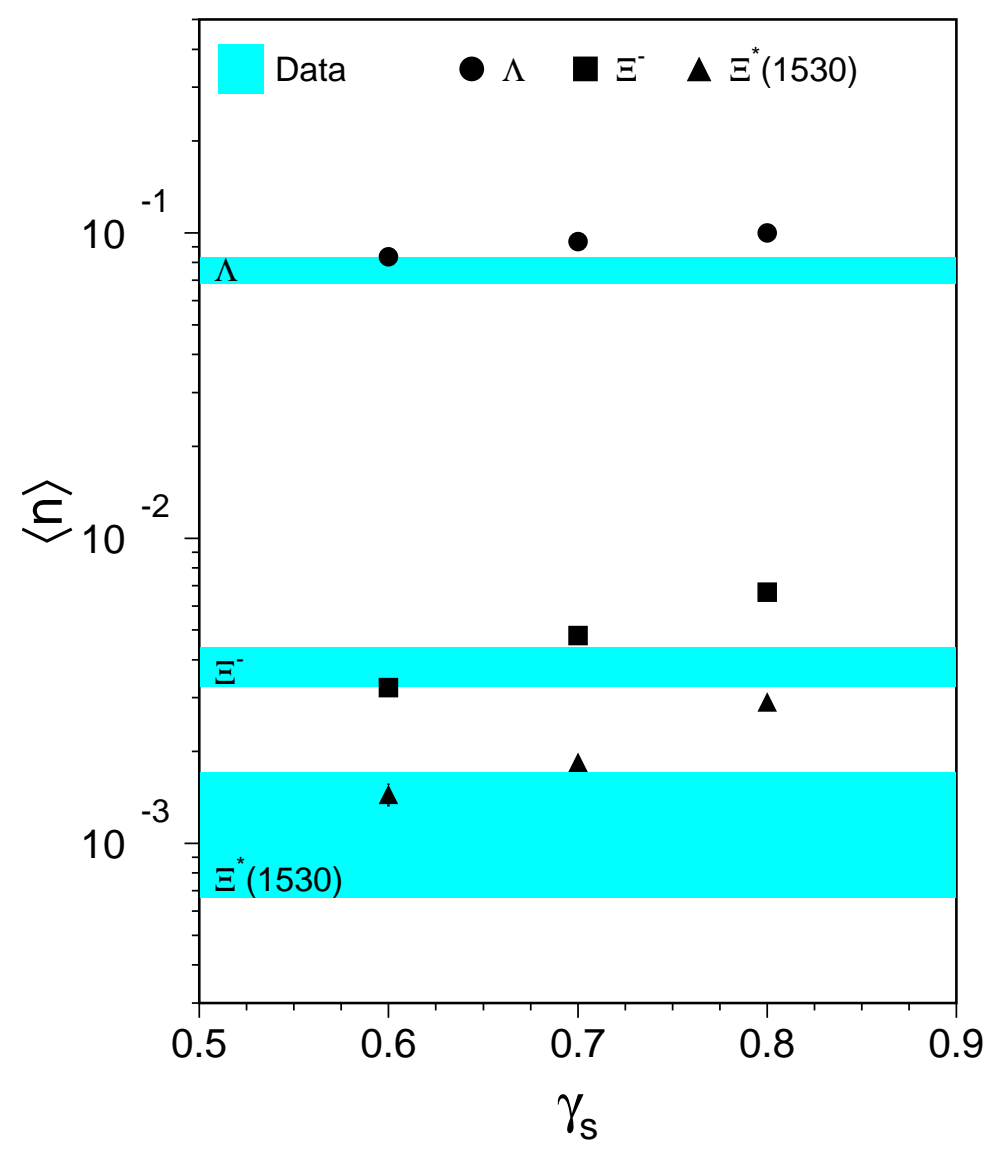

Figure 12: The extrapolated mean multiplicities $\langle n\rangle$ of $\Lambda, \Xi^{-}$and $\Xi^{*}(1530)$ baryons per twophoton event for $W_{\gamma \gamma}>5 \mathrm{GeV}$ (horizontal bars) compared to the predictions of the thermodynamical model as a function of the strangeness suppression factor, $\gamma_{s}$, using as energy density the value $\rho=0.4$ (symbols). 


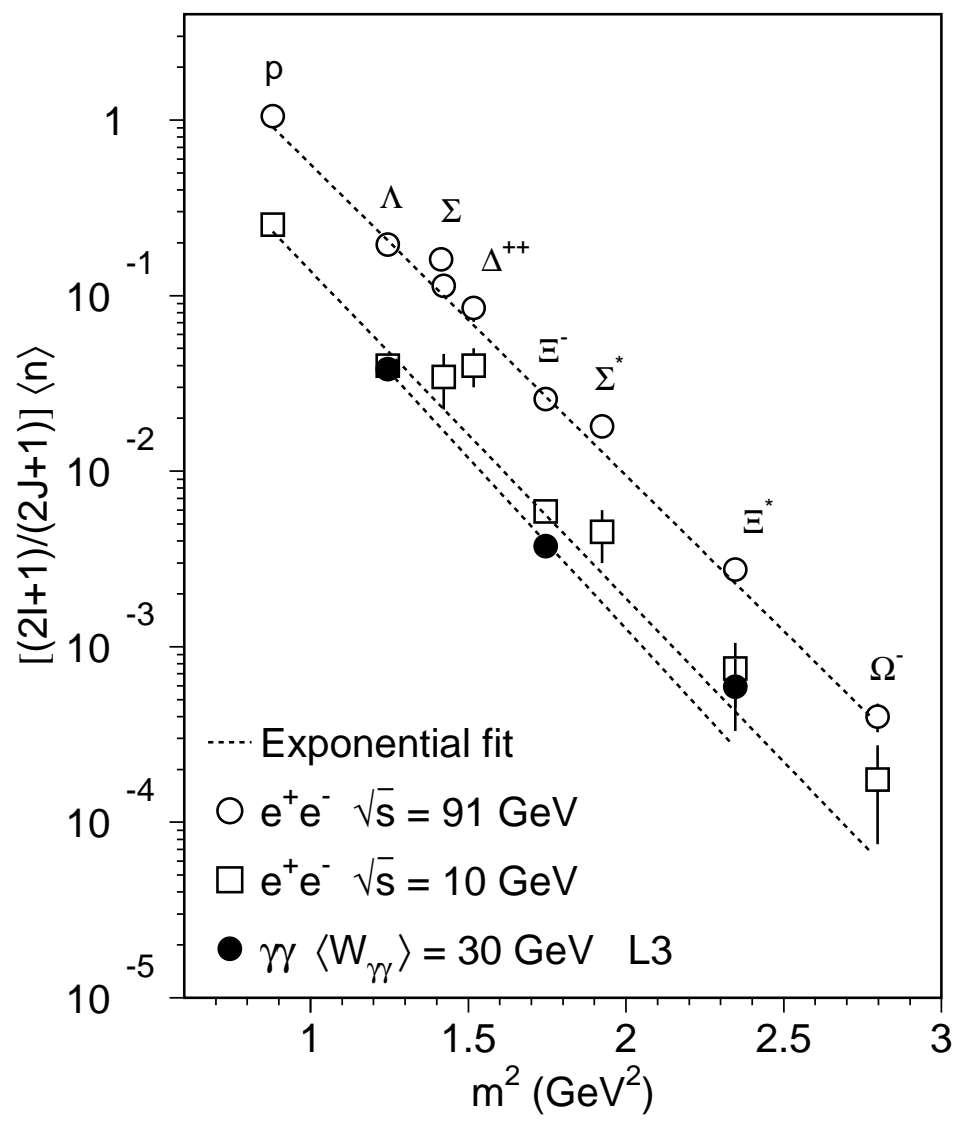

Figure 13: Weighted mean multiplicities $[(2 J+1) /(2 I+1)]\langle n\rangle$ as a function of the square of the baryon masses measured in two-photon reactions (solid circles) together with the data obtained in $\mathrm{e}^{+} \mathrm{e}^{-}$collisions at $\sqrt{s}=10 \mathrm{GeV}$ (open squares) and $\sqrt{s}=91 \mathrm{GeV}$ (open circles). The lines correspond to the fits described in the text. 


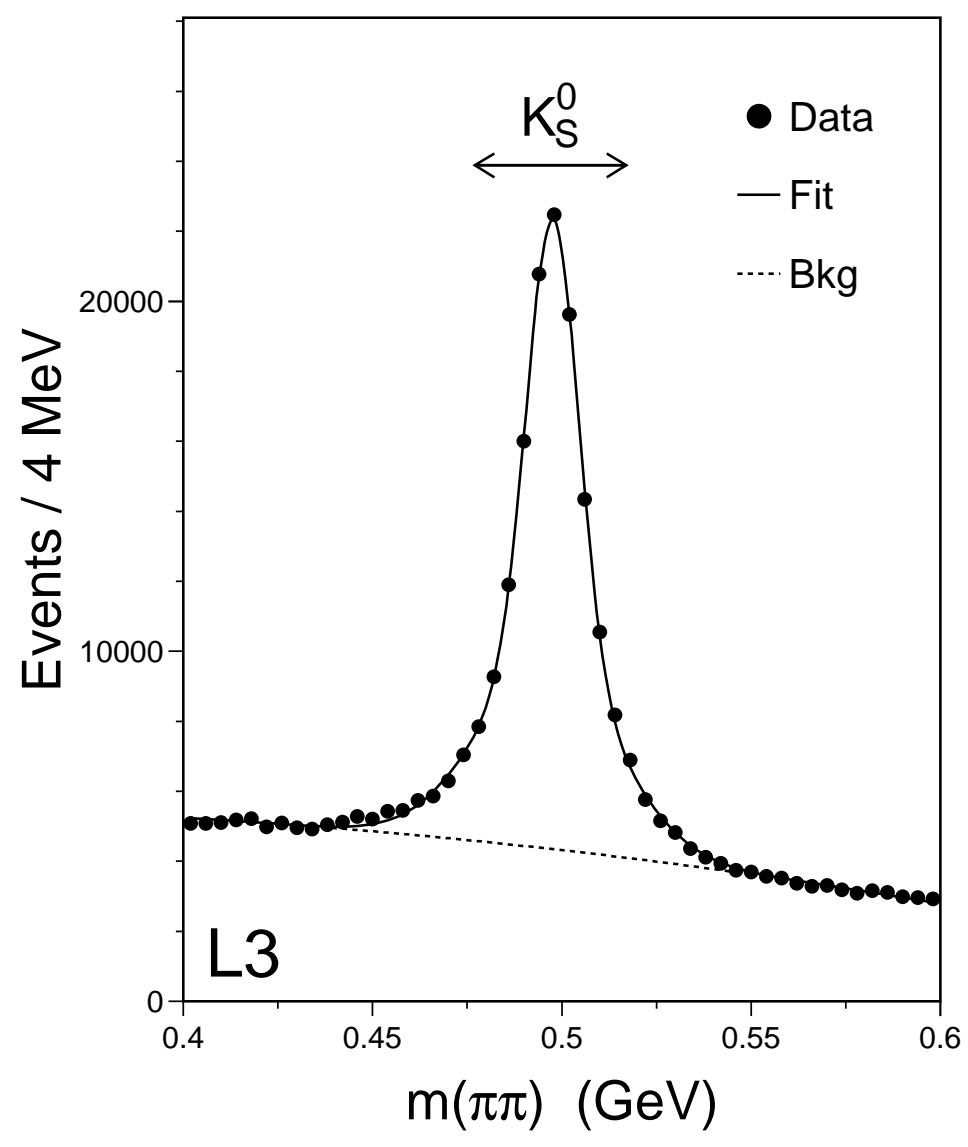

Figure 14: The effective mass of the $\pi \pi$ system. The signal is modelled with two Gaussian functions and the background by a second-order polynomial. About $140000 \mathrm{~K}_{\mathrm{S}}^{0}$ candidates are found in a $\pm 20 \mathrm{MeV}$ window around the central value of the peak and used in the search for the $\theta^{+}$pentaquark. 

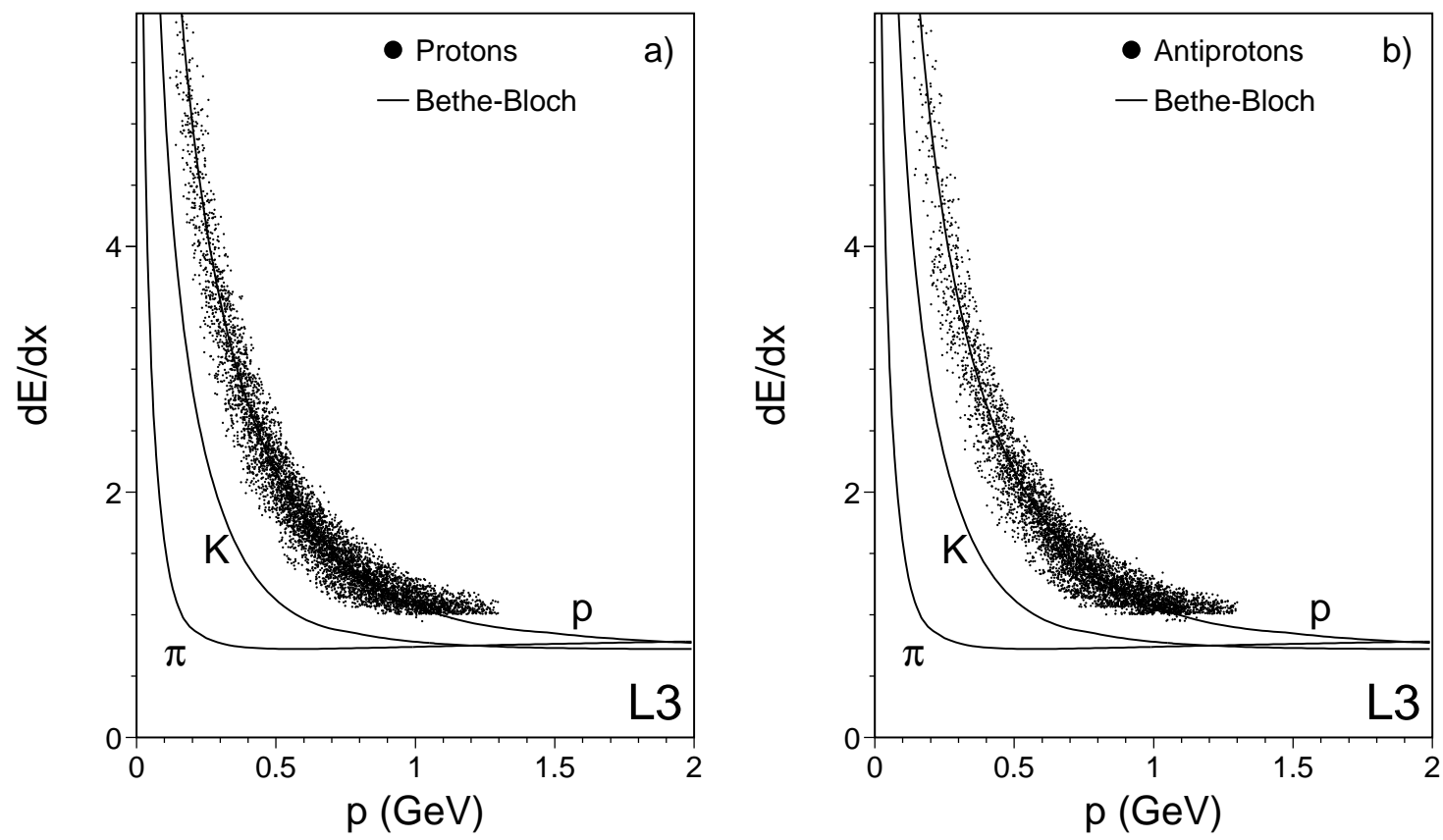

Figure 15: The $\mathrm{d} E / \mathrm{d} x$ measurement as a function of the momentum for the selected a) protons and b) antiprotons, together with the calculations of the Bethe-Bloch formula for protons, kaons and pions.
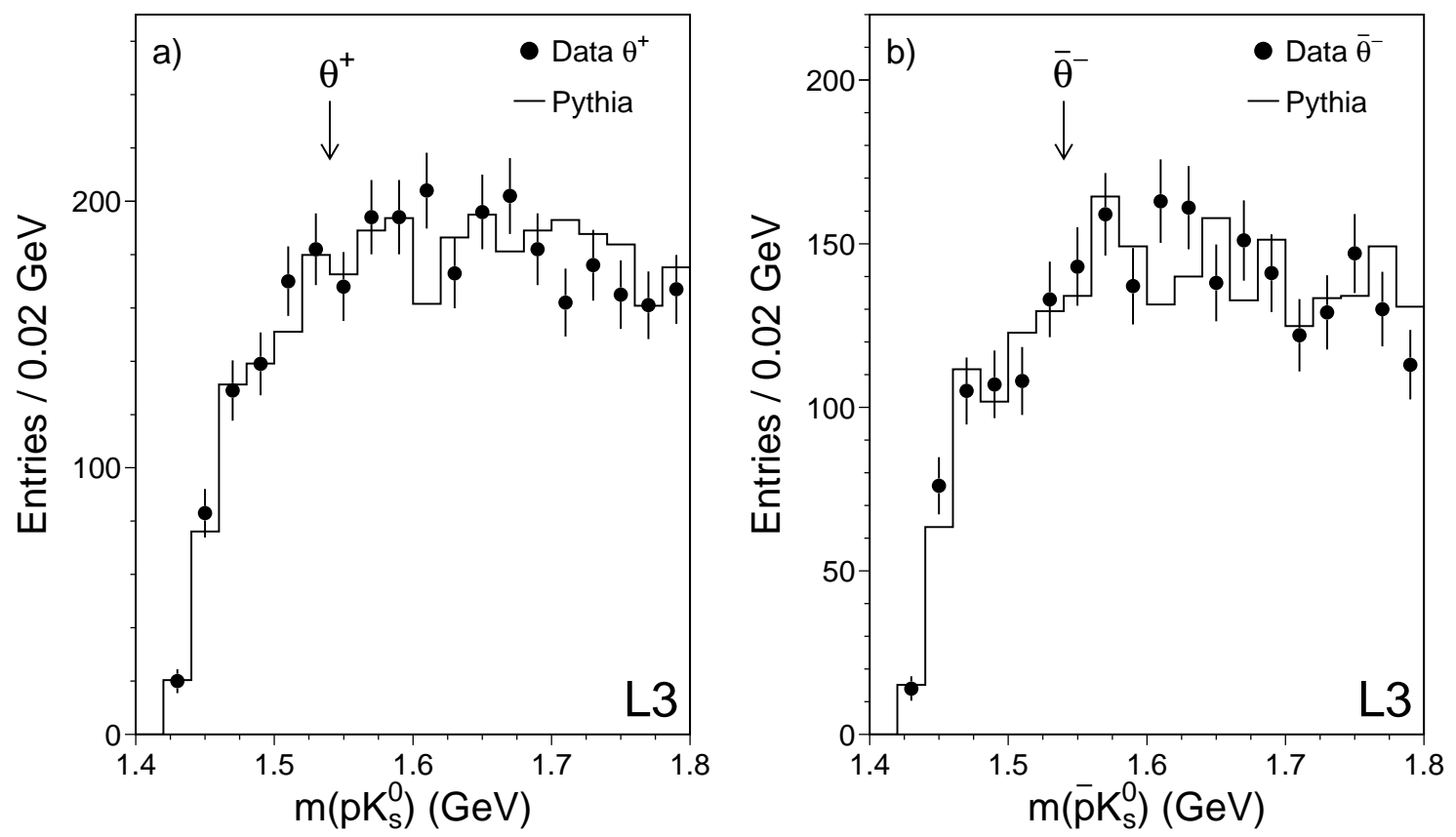

Figure 16: The mass of the $\mathrm{pK}_{\mathrm{S}}^{0}$ system, $m\left(\mathrm{pK}_{\mathrm{S}}^{0}\right)$, for a) protons and b) antiprotons together with the predictions of the PYTHIA Monte Carlo. The arrow indicates the position of the expected $\theta^{+}$signal, around $1.54 \mathrm{GeV}$. 


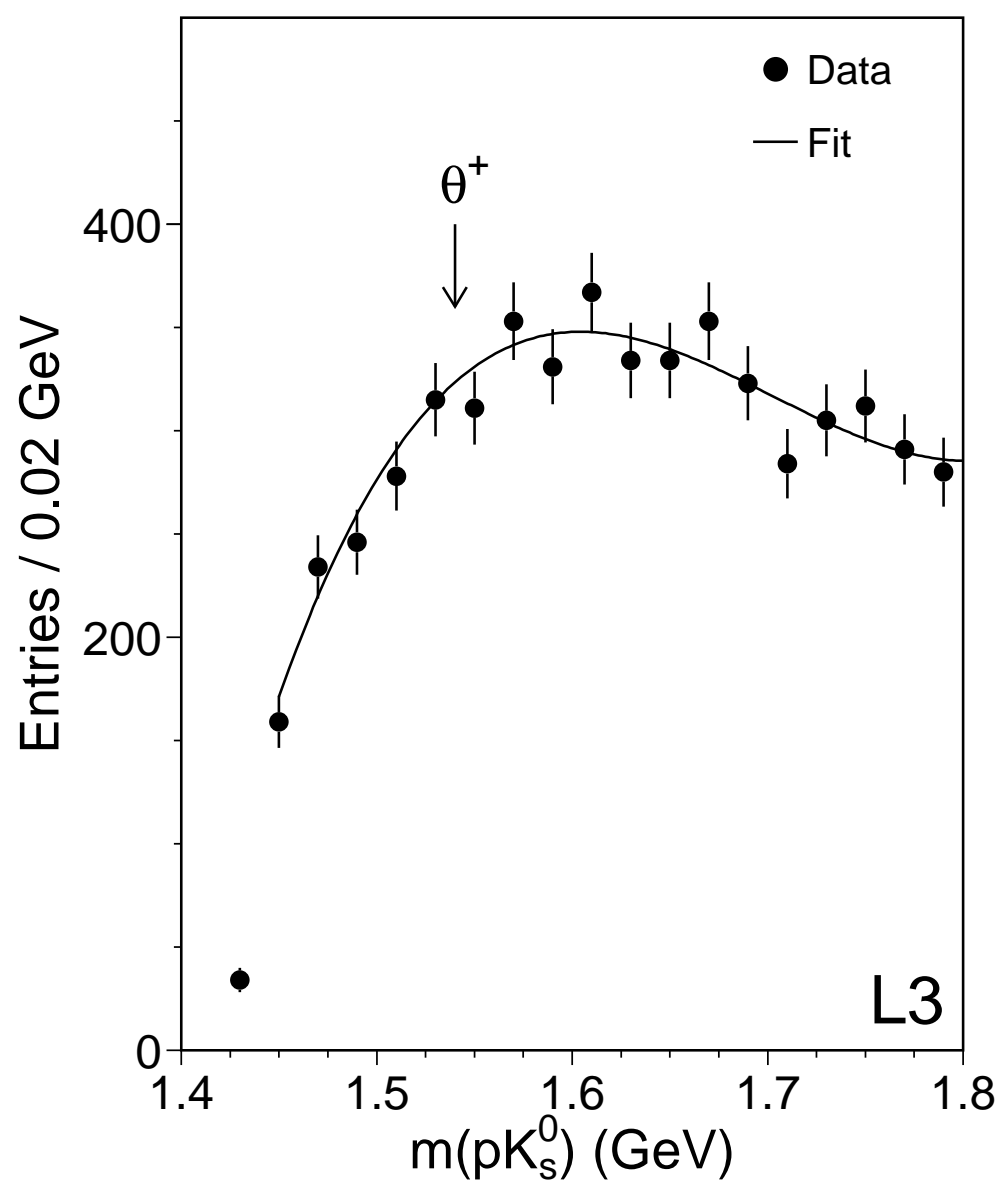

Figure 17: The mass of the $\mathrm{pK}_{\mathrm{S}}^{0}$ system, $m\left(\mathrm{pK}_{\mathrm{S}}^{0}\right)$, for protons and antiprotons combined together with the fit result. The arrow indicates the position of the expected $\theta^{+}$signal, around $1.54 \mathrm{GeV}$. 